\title{
Improvisation et Parrêsia : Des pratiques politiques de soi
}

\section{Fabien Jean-Georges Granjon}

\author{
«Mieux vaut improviser que prévoir le pire. » \\ "L'envers est dans le free. » \\ Bernard Lubat
}

« Nous n'avons fait qu'improviser. Partout. Dans notre rapport aux autres, au politique, au public, suivant ce que l'on avait envie de dire, en fonction des besoins du moment. » Laure Duthilleul

«Donnez tout au moins la parole à la minorité de vous-mêmes. » « L'expression est pour moi la seule ressource. La rage froide de l'expression. » Francis Ponge, Le parti pris des choses

« Tout jadis. Jamais rien d'autre. D'essayé. De raté. N'importe. Essayer encore. Rater encore. Rater mieux ... . Ou mieux plus mal. Rater plus mal encore. . . Rater mieux plus mal maintenant. » Samuel Beckett, Cap au pire

Le présent article est né d'une rencontre avec Uzeste, étonnant village du Bazadais (Gironde, France) qui a vu naître le poly-instrumentiste Bernard Lubat. Après avoir participé, au mitan des années 1960, à l'orchestre de Jef Gilson, au Double-six, ou encore au Swingles Singers, ainsi qu'à de nombreuses autres formations de jazz, de variétés et de musiques classique et contemporaine, Lubat va fonder, en octobre 1976, dans le sillage de la création des collectifs New Thing auto-gérés, ${ }^{1}$ la première Compagnie Lubat. Un peu par hasard, il décide, en août 1978, de venir jouer dans son village natal, en compagnie de Michel Portal et, finalement, de revenir s'y installer (Granjon). Uzeste prend alors le relais des expériences free parisiennes. Ce nouvel ancrage territorial invite Lubat à développer une autre forme de pratique artistique expérimentale, ancrée dans une histoire sociale et politique régionale. Le défi spontanéiste des premiers temps laisse alors la place à une gageure d'une autre nature : mettre en relation les propositions esthétiques du jazz, de la musique contemporaine et de l'improvisation libre, avec une culture locale, afin de réinventer un rapport sensible et politique à la ruralité communale. Lubat et Cie montent notamment un festival, les hestejadas de las arts_- "Grandes fêtes des arts » en gascon-, dont l'une des caractéristiques est de mêler des formes de mobilisation à la fois sociales, théoriques et esthétiques. Au-delà de ce rendez-vous estival, c'est évidemment toute l'année, depuis maintenant quarante ans, que se forgent, à Uzeste, des subjectivités en résistance qui tentent d'ouvrir des espaces d'autonomie. Depuis 2015, nous enquêtons donc pour tenter de rendre compte de ces mouvements de formation de sujets critiques dont l'originalité tient à la combinaison de politiques de la pensée, de l'action et du sensible au sein de laquelle l'art joue évidemment un rôle central.

En tant qu'il est critique, l'art tente en effet de lever le voile de la mise en acceptabilité d'un monde aux ordres qui ne dit pas toujours son nom. Il est en cela un « dérangement de l'ordre où nous étouffons » (Bataille 25). Plus encore, il esquisse des lignes de fuite imaginaires, prend ses distances avec la « réalité » et fait œuvre de "vérité » en désajustant momentanément les correspondances entre des façons d'être, de penser et d'agir et les nécessités présentes auxquelles elles correspondent. En d'autres termes, l'art véritable contribue à mettre en énigme le quotidien-à l'instar du travail des sciences sociales, mais depuis une perspective propre ${ }^{2}$-à changer les consciences, mais aussi à travailler les sensibilités et les imaginaires dans un sens qui vise à éloigner les sujets sociaux de leurs rapports aux mondes habituels : " négation déterminée de la société déterminée » (Adorno, Théorie esthétique 299). Cet éloignement peut épouser différentes modalités, allant du mouvement brownien de l'intranquillité à l'élan de révolte, en passant par la randonnée divertissante ou encore la virée spectaculaire. Son efficience propre - un doute sur l'ordre du monde et une mise en marche—reste incertaine et, quand elle semble avoir eu quelque conséquence, elle n'est évidemment jamais la cause sans reste de l'impulsion libératoire.

Cette recherche d'une efficace perturbatrice se trouve également au cœur de l'improvisation, art de l'éphémère et du pari dont le principe est d'expérimenter des intervalles (Badiou), c'est-à-dire de faire l'expérience de situations où s'ouvrent des possibles non prévus. L'improvisation exprime une poétique de la rupture (Mansour), ébauchant « non l'irréalisable, mais l'irréalisé » (Lachaud 26), où se manifeste, se donne à voir, à sentir et à entendre, l'exercice d'une liberté plus ou moins audacieuse. "L'improvisation, affirme Bernard Lubat, c'est prendre le maquis »; manière de signifier qu'il s'agit de résister en s'éloignant des espaces de consentement qui réifient, ainsi qu'en travaillant son opacité, son équivocité. À Uzeste, l'improvisation est ainsi considérée comme un art « à vivre » qui, s'il n'est pas l'organisateur principal du changement, peut en être néanmoins, considère-t-on, l'un des ingrédients. En certains cas, il peut même s'avérer essentiel : là où, par exemple, les critiques sociale et théorique ont peu de chance de 
pouvoir jouer un rôle premier dans l'armement et l'expression des révoltes, dans la mesure où les conditions de possibilité de leur existence et de leur ascendant peuvent ne pas être réunies (censure, dépolitisation, résignation, faible niveau d'alphabétisation, etc.).

L'improvisation nécessite des individualités singulières-il s'agit de « sonner comme personne » (B. Lubat), i.e. aussi « en tant que personne »-mais le produit de leur rencontre est toujours une émergence pour partie inattendue en ce qu'elle dessine un commun qui ne se confond pas avec la somme des singularités individuelles dont il est issu. L'improvisation dessine en cela des politiques qui font travailler ensemble les différences individuelles dans une action conjointe mise en publicité. L' « identité » de chacun constitue alors la matière première à des actions communes qui la transcendent et font retour sur elle en la certifiant nécessaire tout en démontrant qu'elle ne saurait constituer, seule, le principe de l'agir-ensemble qu'elle a contribué à faire émerger. « D'ici d'en bas » (B. Lubat), l'agir improvisé est donc aussi ce par quoi se forge un sujet politique, lequel ne saurait seulement se résumer à l'individualité idiosyncrasique (ou à l'identité atavique) qui se trouve pourtant à son fondement. L'improvisation se présente ainsi comme une « éduc'action » qui fait de l'agir-ensemble la clé essentielle de la subjectivation et de la construction politiques (Tassin). Nous y voyons également, et complémentairement, une forme de parrêsia, c'est-àdire un travail éthique permettant d'aller à la découverte de soi et de reconnaître le rôle que l'autre joue dans le fait que le Je(u) advienne.

\section{Impro : Qu'es aquò ?}

L'improvisation telle que nous l'abordons, ici, peut être décrite en première approximation comme un acte immédiat de création musicale. Sa complexité et son ambiguïté tiennent à ce qu'elle est un processus : une partition intérieure (Siron) virtuelle actualisée par ce qui ressemble à une forme d'écriture automatique ou de libre association (le jeu) ; mais elle est aussi le résultat de ce processus, lequel se manifeste par un assemblage de sons donné à l'écoute. L'improvisation se présente donc comme une sorte de composition instantanée (e.g. Jerrold Levinson in Canonne), immanente, parfois préméditée et préparée, mais dont une large part-tant dans ses aspects processuels qu'en termes de résultats-relève d'un ajustement pratique à la situation dans laquelle se trouve le musicien qui improvise : « En ceci, sa nature, comme celle de la musique en général, est foncièrement éphémère ; sa réalité est celle de l'exécution. II peut exister des documents relatifs à cette exécution : partitions, enregistrements, chroniques, souvenirs, mais ils ne font que l'anticiper ou la rappeler » (Bailey 152). Celle-ci dépend de paramètres multiples par lesquels l'artiste se laisse affecter et avec lesquels il doit composer : humeurs personnelles, jeu des partenaires, contexte, public, salle, sonorité, etc.

Rester dans une qualité d'improvisation, c'est être toujours en changement. C'est ne pas arrêter ses idées, ne pas arrêter ses pratiques. On a tous une marmite dans laquelle on pense avoir des trucs qui peuvent servir, déjà travaillés, vécus dans le passé, mais Ô malheur, ils n'arrivent pas ça sur scène. Il y a un nouvel objet qui se fabrique devant soi, devant le spectateur et qui n'appartient à personne. L'improvisation, c'est Benedetto, c'est l'acteur-loup sur une des pistes de Scatrap [album de la Compagnie Lubat], l'acteur qui n'est pas le chien apprivoisé avec ses techniques de chien savant, mais qui échappe, qui s'échappe, qui part dans l'indicible. Être improvisateur, c'est accepter de devenir loup, c'est-à-dire la possibilité d'être sauvage, préférer se bouffer la patte que d'être pris au piège. (Jaime Chao, informaticien multiinstrumentiste de la Compagnie Lubat, avril 2016)

Accomplissement pratique, l'improvisation peut respecter assez largement les normes de la construction musicale (mélodie, séquences harmoniques, rythme, mesure, tempo métronomique, justesse, etc.), s'inscrire dans un style ou un idiome particulier (swing, be-bop, etc.) et répondre à des cadres d'action préformalisés et attendus-paraphrase, phrase-chorus, libre variation (Gerber, « Le Cohelmec » 168). A contrario, elle peut également s'en détacher largement pour aller vers des formes plus libres et " non-idiomatiques ${ }^{3}$ » (Bailey 13), tendues vers " l'attente de l'inattendu » (Quan Ninh 62). L'improvisation s'étire donc sur un continuum allant d'expressions stylisées dans un langage qu'il est possible de reconnaître comme relevant d'un genre de codification singulier-c'est-à-dire appartenant à une suite d'occurrences que l'on retrouve généralement liées les unes aux autres dans une forme de jouage-jusqu'à des formes d'expressivité débridées, plus expérimentales, difficilement codifiables, qui exigent toute licence 4 en musique, qui cherchent à créer « le son de la surprise » (Bailey 65), l'inentendu, ainsi qu'à explorer, par l'expérience, " comme toutes les possibilités d'écriture en même temps, [i.e.] potentiellement toutes les esthétiques » (Quan Ninh 33).

Mon investissement à Uzeste a été une réaction au théâtre dans lequel j'étais à l'époque, en 1984, un théâtre public subventionné intello de grande qualité, mais il y avait quelque chose qui me gênait, mais sans avoir, à l'époque, la capacité d'analyser ce qui coinçait. C'était un ressenti. Je jouais dans Terre étrangère d'Arthur Schnitzler au Théâtre des Amandiers, dans une mise en scène de Luc Bondy ; c'était passionnant, mais il y avait une lourdeur que je trouvais louche dans l'opulence des décors, de la mise en scène, du 
travail même d'acteur, dans le fait que tout le monde courait pour venir voir ce spectacle. Je me suis investie à Uzeste, parce que la manière de faire était très différente, plus intuitive, plus expérimentale et nettement moins mondaine. Ça me ressemblait davantage. On improvisait, là où d'habitude il fallait des répétitions, passer des heures autour de la table, etc. L'improvisation c'est du culot, de la créativité et accepter de te planter. C'est aussi beaucoup de travail, même si l'on joue toujours son histoire. (Laure Duthilleul, comédienne, scénariste, réalisatrice, ex-membre de la Compagnie Lubat, avril 2015)

Je sais ce qu'ils me disent les chanteurs... ils me disent qu'ils adoreraient faire ce que je fais, d'avoir cette liberté. Évidemment ils pourraient le faire, mais ils ne le font pas parce qu'ils n'auraient pas de dates avec ça. Donc ils continuent à être dans « le style ". ${ }^{5}$ Le style c'est un truc marketing, on est envahi par le style ; il faut faire du style. Faire du style, c'est faire des choses avec lesquelles ils peuvent décrocher un Marciac ou une scène nationale. Ils le font bien, mais ils n'innovent pas, ils n'ouvrent pas des nouveaux possibles, ils reproduisent. Sauf que ça sert à quoi de faire ça ? Nous, à Uzeste, on a la prétention de faire un art qui émancipe l'artiste et aussi les spect'acteurs. On gagne rien avec ça si ce n'est, et c'est énorme, notre liberté. (Fabrice Vieira, guitariste et vocaliste de la Compagnie Lubat, mars 2017)

La compagnie Lubat a joué un rôle important dans cette capacité à improviser. Bernard Lubat a été un élément déclencheur essentiel de mon travail. Dans la rencontre même, au départ, sans même les mots. J'ai travaillé, à un moment donné, dans la Compagnie et les journées de travail, elles étaient improvisées. J'étais libre de faire ce que je voulais, d'installer mes machines comme bon me semblait. Quand Bernard se produisait à l'Estam, j'avais carte blanche pour balancer ce que je voulais. J'avais un tourne-disque et je balançais "Je suis malade" de Lama ou autre chose. J'avais un parcours de boules de pétanque qui allumaient des lampes, qui tombaient dans un escalier, sur une taule. À l'époque je travaillais sur le son, mes installations étaient ciblées là-dessus. Et ma place, je l'ai improvisée, elle n'existait pas du tout. Être libre pour créer. Avec la Compagnie Lubat, on se trouvait dans un bain de création, c'est-à-dire d'improvisation. Je créais dans mon atelier, j'installais à l'Estam. J'ai aussi fait mes premières expos à Uzeste. C'est à cette occasion que j'ai rencontré Meschonnic. Improvisation, sensible, pensée . . . Tout ça se mélangeait. (Patrick Deletrez, plasticien compagnon de route de la Compagnie Lubat, octobre 2016)

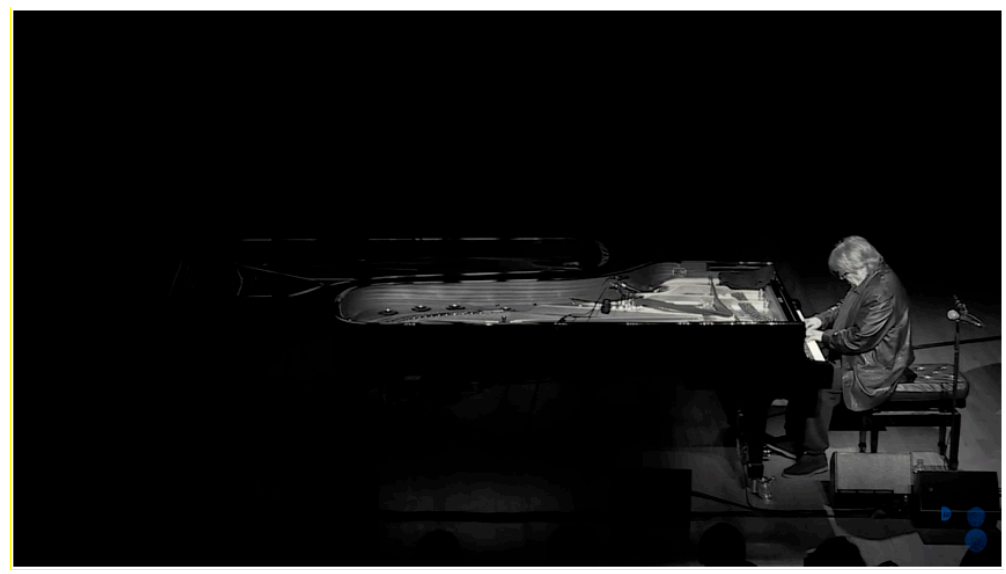

Extrait du film In \& Out - Martial Solal-Bernard Lubat

https://youtu.be/GdjuShqKaxw

Y compris dans ses déclinaisons les plus free, l'improvisation n'est cependant jamais, seulement, différences absolues, mais plutôt différences relatives car elle est indexée à des perceptions changeantes de situations changeantes (Quan Ninh). De surcroît, elle s'arrime à des repères stylistiques et comporte de la mêmeté, des répétitions, des ressassements, des accumulations : «Bien sûr on peut trouver des accointances, on voit bien que je viens du Jazz, mais je continue le Jazz. Je ne récite pas d'où ça vient, je ne récite pas d'où je viens, mais je tente le coup d'où je vais. J'essaie de me rappeler de ce qui n'existe pas encore » (B. Lubat). Quand Lubat joue, pour la énième fois, le standard mingussien Goodbye Pork Pie Hat, ${ }^{6}$ il ne s'agit évidemment pas d'ânonner un blues en fa que l'on connaît par cœur, mais d'actualiser les subtilités d'une composition depuis laquelle peut s'envisager d'infinies inflexions, des commencements, c'est-à-dire des traces, des chemins contrebandiers, une errance qui, comme le suggère Édouard Glissant, est aussi, paradoxalement, " le lieu de la répétition, ${ }^{7}$ quand celle-ci aménage les infimes (infinies) variations qui chaque fois distinguent cette même répétition comme un moment de la connaissance [de soi] » (Philosophie 61-62). On pense évidemment à la musique de John Coltrane et, plus particulièrement, au travail qu'il n'a cessé de mener sur ce standard un peu mièvre de Richard Rodgers et Oscar 
Hammerstein ॥—composé à l'origine pour une comédie musicale de Broadway, The Sound of Music (La Mélodie du bonheur)-My Favourite Things (Gerber, Le cas Coltrane; Arcens ; Imbert) qu'il enregistre pour la première fois sur un album éponyme (Atlantic Records, 1961) et qu'il n'aura de cesse de jouer, de rejouer, parfois jusqu'à la transe, comme sur cet incroyable enregistrement d'un concert qu'il donne en quintet le 2 juillet 1966 au festival de Newport (Last Performance at Newport-Free factory, 2009). Michel Delorme et Jean Clouzet voient dans le travail de Trane, et plus particulièrement dans son rapport à My Favourite Things, " une plongée totale au fond de soi-même, l'exploration méthodique, obstinée, sans espoir parce que sans fin, de ses ressources et de ses possibilités » (Coltrane 22).

Et Lubat de commenter : « L'original de My Favourite Things n'est pas terrible. Coltrane en a fait un monument en archaïsant cette composition qui était initialement maigrement savante. C'est devenu une ritournelle étrange qu'il fait passer de la naïveté à l'indécence, c'est-à-dire à l'incandescence des sens. C'est un morceau sur lequel ils se lâchent, ils s'incitent mutuellement à leur perte. Coltrane et ses musiciens ont compris que les intervalles, ce sont eux, d'abord ce qu'ils ont en eux et qu'ils expriment avec une violence terrible. Ils jouent leur vie, ça ne rigole pas, c'est dramatique. Quand j'improvise, je deviens ex-centrique, je volontarise une démarche qui se projette, qui s'imagine. C'est curieux parce que c'est à la fois se propulser vers le non-savoir, vers le dé-savoir, le dé-se-voir_quand tu t'es bien vu, t'es déçu-vers le non contrôle idéologique - on ne sait pas ce qu'on va dire et ce qu'on va faire, on ne sait pas quelle forme ça va avoir, si c'est bon, si c'est mauvais ... on ne sait pas_-mais on sait qu'il faut qu'on aille dans le "on ne sait pas." C'est dans le "on ne sait pas" que se sait quelque chose. Ça veut dire que d'un seul coup on est coupable d'être capable. Ou capable d'être coupable. Et on produit, comme un enfant qui ne sait pas dessiner: on produit un monde. Un monde crié qui n'est ni celui de la "noblitude" des conservatoires, ni celui putassier de la marchandise : l'abîme de l'exploration-expérimentation entêtée de soi. Sous cet aspect, My Favourite Things est une merveille du genre—il y en a d'ailleurs beaucoup d'autres chez Coltrane_la viande avant les notes, le musicien avant la musique. ${ }^{8}$

Les élans de l'improvisation libre peuvent par ailleurs intégrer une variété d'éléments non musicaux qui ressortissent d'autres domaines artistiques (théâtre, slam, vidéo, etc.), mixant par exemple images et/ou mots : "Les Mosicans ${ }^{9}$ [spectacle de la Compagnie Lubat], c'est la liberté de jouer des mots, de la musique, des sons, des bruits, et peutêtre même des idées " (B. Lubat). À Uzeste, les publics sont mis en contact avec des signifiants fluctuants et incertains, mélangeant des formes à la fois reconnaissables et inédites (Bonnerave). L'improvisation mosicaleconstituée de musique et de mots—s'y appuie sur des repères (chansons, thèmes, « hymnes », citations, jouages, etc.) qui donnent des prises momentanées aux auditeurs, mais ils sont la plupart du temps déconstruits, reconstruits, enrichis, mélangés, etc.

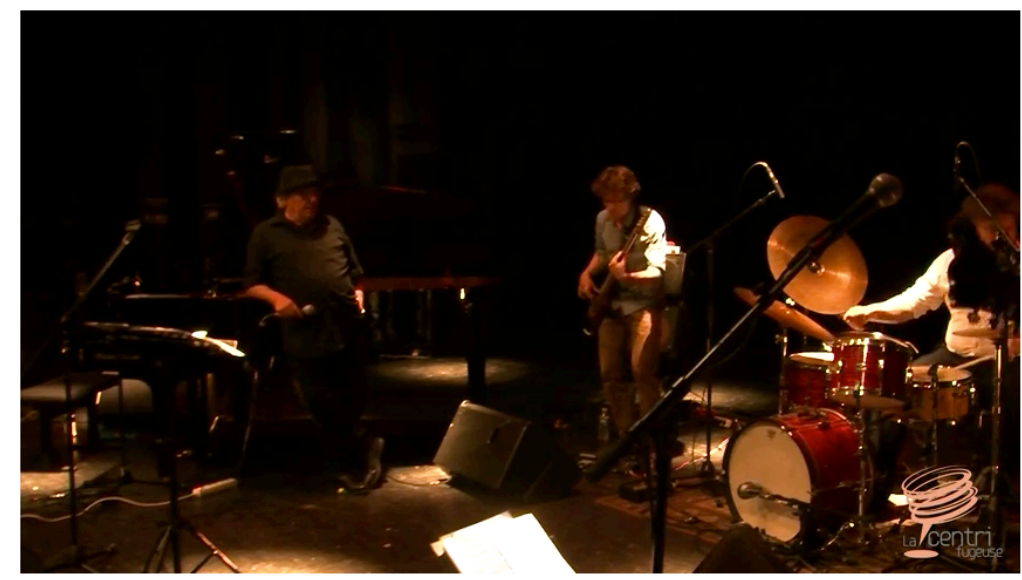

Extrait d'un concert de la Compagnie Lubat à La Centrifugeuse (Pau, France) https://youtu.be/PLINtkSdwx8

Comme le rappelle Denis Laborde, l'émotion qui naît de l'improvisation « n'est donc pas du seul ressort de la trompe d'Eustache, c'est aussi une question d'entendement » (145). L'improvisation est donc " une manière de dissidence » (d'« ici-danse » aurait écrit Lubat), car elle s'appuie sur de « nouveaux rapprochements par croisements, montages ou collisions [qui] défont la syntaxe et les délimitations convenues : jazz moderne, musique contemporaine, avantgarde expérimentale. Une façon de transhumance aussi : hors des enclos assignés, la pensée musicale de Lubat ne cesse de translater modes expressifs et codes formels d'un morceau l'autre » (Moussaron 105). L'improvisation est ainsi créolisation et poïélitique, c'est-à-dire une manière de faire pénétrer l'imaginaire dans la pensée, le politique 
dans le sensible et de faire de cet enchâssement une puissance qui libère certains possibles et appelle à actualiser leurs restes : " une sorte d'hybris qui se révèle, de façon inattendue peut-être, porteu[r] d'un avenir, et signe d'une dynamique imprévisible » (Ménil 220).

À Uzeste, cette construction de l'inattendu est qualifiée de « composition multim(m)édiate ». Toutefois, cette immédiateté « multimédia » d'une « musique sans papier, $100 \%$ improvisée » ne relève pas pour autant d' " heureux hasards » ou d' « heureux bazars ». Elle répond plutôt à une sérendipité ${ }^{10}$ assumée qui se couple à un engagement (une volonté) et fait pièce avec une préparation, voire une certaine discipline : « L'improvisation ce n'est pas le spontanéisme abscons. L'improvisation, c'est un art, ce n'est pas le hasard seulement. C'est une convocation à l'inconscient. C'est une façon de laisser parler ce qui ne parle pas tout seul, ce qui ne va pas de soi » (Lubat in Chemillier). « L'improvisation, ce n'est pas le bordel. Assez vite, j'ai proposé d'écrire les programmes des festivals, d'écrire les synopsis des spectacles. Non pas pour figer les choses, mais pour les faciliter » (L. Duthilleul, avril 2015). Et Jaime Chao de renchérir : "L'improvisation ne vient jamais de rien, elle vient se mettre en contact et en friction avec d'autres pratiques, de pensée notamment, d'organisation de la pensée. Toute cela se met en crépitement et ça fabrique de l'inattendu » (avril 2016).

Comme aime à le rappeler l'Amusicien d'UZ $Z^{11}$ : «On ne s'improvise pas improvisateur ». Improviser demande en effet quelques préalables, s'agissant notamment de la maîtrise des outils de production sonore et scénique, et bien évidemment de l'écoute. Une technique instrumentale ample est un prérequis central puisqu'il s'agit de disposer de potentialités d'expression étendues-une « qualité de réaction » (Jean-Pierre Drouet ${ }^{12}$ in Gerber, " Le New Phonic Art » 200)—afin de se trouver en capacité de traduire, le plus directement et fidèlement possible, une idée ou une sensibilité en une oralité (Nachmanovitch; Ben Sidran définit la musique afro-américaine comme culture orale ${ }^{13}$ ). II s'agit donc d' « avoir la technique de ses fins propres », étant entendu que « bien jouer, n'est pas jouer avec la technique la plus accomplie, mais mettre dans sa musique la plus grande charge émotionnelle possible » (Gerber, « Le Cohelmec » 184), c'est-à-dire y placer ce qui permet de faire collectif, d'inciter l'autre, de le mobiliser (nous y reviendrons) : «C'est énorme d'arriver avec tout son background, que tous les jours tu empiles et de monter sur scène pour tout remettre en jeu. C'est grâce à ce background que tu es capable d'improviser et que tu es capable d'inventer, de rêver tout, de n'être pas d'accord avec ce que tu dis, d'inviter l'autre » (Lubat, mars 2017). Si comme le suggère Lê Quan Ninh, « ne pas savoir, c'est commencer à improviser » (8), il faut saisir que l'ignorance ou la méconnaissance dont il est ici question ne porte pas sur un manque de savoir ou de savoir-faire, mais sur l'incapacité à prévoir ce qui sortira au final de l'improvisation. Improviser, c'est donc doublement parier sur les résultats esthétiques et sur le processus qui en est à l'origine : «II faut beaucoup improviser pour devenir libre. Ne jamais inventer le même parcours entre des pierres posées. La liberté, on ne la gagne pas, on l'affronte. C'est un exercice qui t'oblige à être au maximum de tes capacités et des capacités ça se travaille : travailler l'instrument certes, mais surtout autre chose ; travailler l'histoire, se comprendre, comprendre les autres dans un même mouvement » (F. Vieira, avril 2016).

Plus précisément il s'agit de croire en la viabilité de ce processus, i.e. conjecturer son caractère vivant et erratique : avoir confiance en la « disponibilité de l'étant pour toutes sortes de migrations possibles » (Glissant, L'imaginaire 37) et donner quelque créance à la fragilité, à la dérive_-presque au sens situationniste du terme. II s'agit d'expérimenter par la pratique musicale une pensée théorique de la totalité et du Tout-monde. L'improvisation se fait donc drive, dans l'acception créole du terme — « la disponibilité, la fragilité, l'acharnement au mouvement » —Glissant, L'imaginaire 37), mais aussi poétique du flux (le drive du batteur) qui, répétons-le, fait toujours cas de l'épreuve des situations : "Accepter les circonstances présentes c'est accepter le mouvement permanent qui les anime. Le travail d'improvisation est de n'y rien figer, mais d'entretenir ce mouvement par le fait même de s'y glisser » (Quan Ninh14). L'œuvre improvisée ne peut être ainsi qu'à l'œuvre ${ }^{14}$ dans la mesure où elle ne saurait quitter le domaine de « l'en train de se faire ", à un train d'enfer. L'improvisation se fait ainsi digenèses (Glissant), elle est mouvement de création qui n'en finit pas. De nature imprédictible, elle se compose de commencements « qui fluent de partout comme des fleuves en errance » (Glissant, La Cohée 37). Elle produit des intervalles sensibles qui permettent d'après Lubat « de laisser respirer la pensée et l'imaginaire » (février 2016). De nature inchoative, l'improvisation est un art de l'enfance — " garder l'enfant libre en nous » —qui s'escrime à retrouver un peu de cet enchantement qui accompagne la découverte du Je et de cette jubilation du jeu de la découverte :

De l'enfance cette part perceptive où le monde et les autres découvrent par touches, entre rêve et réalité, où les choses ne sont pas encore nommées mais sont pure présence dans leurs formes et leurs volumes, dans leurs textures et leurs couleurs, dans leurs capacités à créer des ombres qui se transforment et bougent au gré de la lumière qui les frappe. Alors l'improvisation libre comme une enfance de l'art, une enfance qui se trouve pourtant dans l'extraordinaire complexité des relations, dans la technicité mise en œuvre, dans la virtuosité sensorielle nécessaire. Ce serait comme si cette pratique permettait de devenir adulte-dans la maîtrise des moyens_-en se rapprochant au plus près du cœur de l'enfance—dans l'origine des sensations. Improviser, ce serait jouer comme au premier jour. (Quan Ninh 29) 
À l'habileté manuelle sonore qu'appelle l'improvisation fait naturellement écho un autre impératif qui relève, lui, d'une capacité d'écoute, c'est-à-dire de l'aptitude à saisir et à s'imprégner des éléments (sonores) extérieurs notamment produits par les partenaires de jeu, mais émanant aussi éventuellement d'autres sources (e.g. des auditeurs censés participer à ce qui doit advenir sur scène). Débriefant une prestation scénique (Improvisions poétiques) effectuée lors de l'Uzestival 2016 du nouvel an, en compagnie de Juliette Kapla, Méryl Marchetti et Michel Ducom, Jaime Chao précise ainsi : " J'ai essayé de trouver des mots qui rebondissent sur l'instant, des mots qui rebondissent sur les gens qui sont là sur scène et dans la salle dans laquelle je suis resté avant de monter sur scène, pour prendre le pouls. Je compose avec l'environnement et après j'amène vers les choses qui m'intéressent, c'est-à-dire le "malpolitique" : comment nous les artistes on doit titiller et être titillés par nos titillements » (avril 2016). Cette compétence perceptive est également la déclinaison pratique nécessaire d'une disposition plus générale ${ }^{15}$-Drouet parle à l'égard d'« une constitution, morphologique » (in Gerber, « Le New Phonic Art » 200)—dont on pourrait affirmer qu'elle est une manière de s'engager dans la situation, de répondre à la hauteur de ses possibilités, penchants, talents, envies, sentiments, désirs, etc., de façon (im)pertinente, en étant sensible à la présence de l'autre et de rendre l'autre sensible à la sienne :

Si mon outil en tant qu'improvisateur est l'ensemble de mes perceptions, outil qui se concrétise en quelque sorte dans le médium qu'est l'instrument de musique, tout mon travail sera d'étendre mes capacités perceptives ou tout au moins d'en considérer les limites et les comprendre mieux. [ . . . ] Plus ce travail est ouvert, c'est-à-dire plus le réel est découvert, plus ma faculté de le découvrir est augmentée. Ainsi, ma liberté perceptive ne s'arrête pas là où commence celle des autres, mais elle s'en enrichit sans cesse. (Quan Ninh 10)

L'exigence « improvisatoire » est ainsi « mouvement perpétuel d'échanges » (Quan Ninh 37), un rapport à l'altérité. En cela, elle est proche de celles que définit Glissant s'agissant de la Relation et de la créolisation (2009), lesquelles relèvent de l'éthique, c'est-à-dire de la manière d'être et d'agir avec les autres.

L'improvisation, c'est l'art de la relation. Une relation qui permet d'affronter son propre soi tout en étant avec les oreilles et les yeux avec les autres et de proposer à l'autre, toi, tout en écoutant ce que les autres ont à te proposer. C'est d'être dans cette relation de jouer ses différences. L'improvisation n'est pas tant la liberté, que la libération de soi ${ }^{16}$. C'est une révolution personnelle jouissive qui libère et fait jouir. Et ça, ça fout la merde. (F. Vieira, avril 2016)

C'est par l'autre, par ses différences, son ambiguïté, sa complexité que peuvent se faire jour des situations inédites, susceptibles d'ouvrir à des possibles non envisageables en dehors de pouvoir en faire l'expérience : " arriver à être libre avec la qualité de l'autre ». "Plus on joue, et plus on s'enfonce en soi-même, mieux on tire parti de ce qu'il y a à l'intérieur de nous, mieux on tire parti de ce qu'il y a dans le monde. . . . Improviser, . . . c'est être dans le moment, au beau milieu de toutes les relations possibles » (Parker). C'est par la Relation que les normes se relativisent, les inhibitions se dépassent, la matière à penser, à sentir et à faire circuler, que se révèlent « les lieux-communs dont nous devinons entre nous le partage » (Glissant, Philosophie 72).

La relation à l'autre, c'est cela, c'est le doute. Et moi, j'avoue que je reconnais plutôt que la musique que je préfère, c'est celle qui est transportée par ce doute, qui est dans cet endroit " entre », entre lui et elle, entre lui et l'autre, entre Noir et Blanc, entre vrai et faux, entre gentil et méchant. À partir du moment où on arrive à convoquer cet espace, qui est un espace physique et psychologique, et donc politique, il se passe du point de vue esthétique des choses qui me passionnent en tant qu'amusicien. D'un seul coup arrivent des commencements nouveaux, d'autres commencements qui n'étaient pas prévus au programme, dont il n'était pas prévu qu'ils commencent, des commencements qui viennent, encore une fois, de partout, et souvent d'ailleurs, du bout du nez, qui ne viennent ni des hauteurs, ni des profondeurs. (Lubat in Chemillier) 


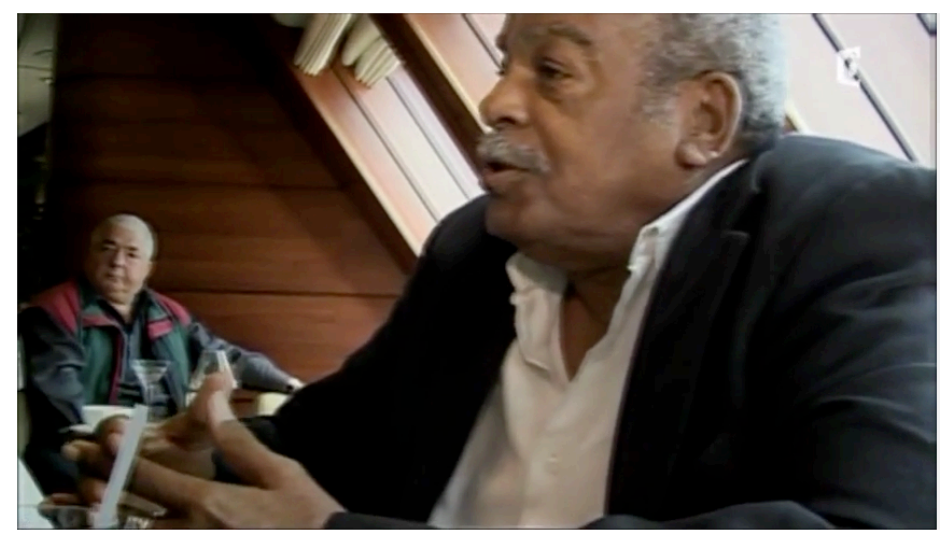

« Être et créolisation » : extrait d'un entretien avec Édouard Glissant - France Ô https://youtu.be/Zt7SL_IRIBw

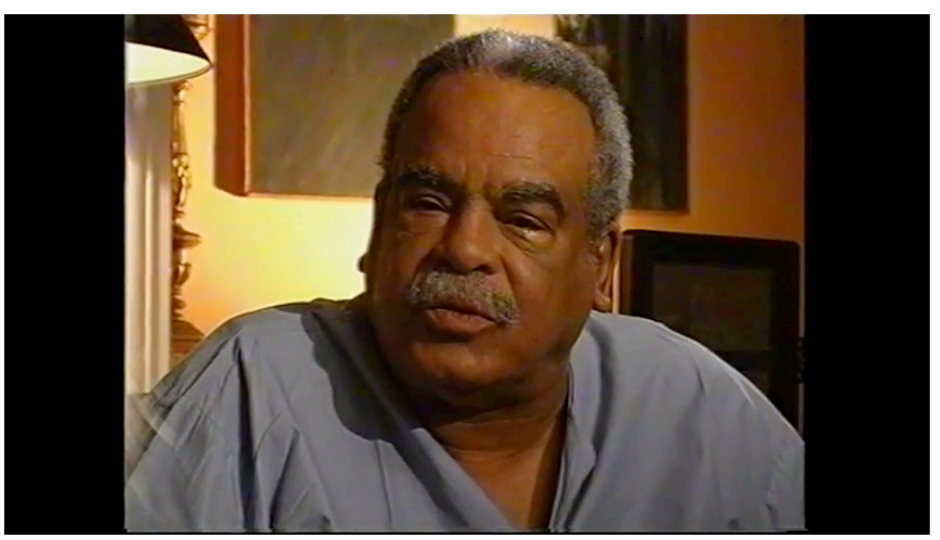

« Relation » : extrait d'un entretien avec Édouard Glissant - TV5 Monde/RFO

https://youtu.be/ys1C5g6Tpgs

Improviser, c'est ainsi « porter attention à la présence de chacun, vraiment, dans un affût bienveillant, parce que cet instant en devenir fuit et fuit à jamais. Parce que tout à l'heure il n'y aura plus aucune trace de cet instant, plus aucune trace de la chair et de la grâce de cet instant » (Quan Ninh 12).

Qui a commencé ? Quand on se rencontre avec l'autre, qu'est-ce qu'on en fait ? Qu'est-ce qui se passe ? On se met au service de qui, de quoi ? Où est-ce qu'on est libre ? Qui a tort, qui a raison ? Dans la musique contemporaine occidentale, une musique qui n'a pas de style, de prédéfinition, une musique qui n'existe pas, qui part de rien, simplement de l'un et l'autre, sans but précis de terminaison, qu'est-ce qu'il peut se passer dans ces conditions où c'est l'incertitude de l'autre? Et comment s'articulent et s'échangent des sons, des phrases, des textures, des alphabets, des syntaxes sonores, des "badabudu, ha, boudé, huuuuu" ? Cela semble très difficile à faire. Chacun, semble-t-il, craint de perdre, pour ceux qui croient au Père Noël leur âme, pour d'autres du pognon, une nana, un pouvoir. Enfin, il y a toujours quelque chose à perdre. Et ce n'est pas faux ! C'est là que cela se passe à la condition d'accepter de perdre, de savoir perdre. Et peutêtre que le savoir, c'est "perdre" » (Lubat in Chemillier). " Ça n'arrête pas de commencer, ça n'a jamais commencé et ça n'a jamais fini, c'est "entre" que ça se passe. C'est pour ça que le jazz se "délibère" dans tous les sens. (Lubat in Chemillier)

Et de se poser nécessairement cette question essentielle : « Comment être soi sans se fermer à l'autre, et comment s'ouvrir à l'autre sans se perdre soi-même ? " (Glissant, Introduction 23). De fait, l'improvisation se présente comme une pragmatique de la rencontre orale qui privilégie finalement le faire sur le résultat de ce faire, ${ }^{17}$ « le jeu sur le joué » (Gerber, « Le Cohelmec »180), la « primauté du processus de production sur le produit et de l'événement sur l'objet esthétique ${ }^{18}$ » (Gerber, « Le New Phonic Art » 201).

L'accent est mis sur l'improvisation comme processus de production et non pas comme produit. L'importance est dans le faire, dans un certain travail pris dans la dimension du temps : autrement dit,

l'improvisation ne peut être considérée comme un objet de contemplation, dont on puisse faire le tour [...], 
elle doit être pratiquée. Du point de vue du spectateur, c'est l'activité humaine concrète (Marx, Engels) qu'il convient de viser et non la musique-chose dont elle accouche ; il y a refus de s'aliéner dans son travail. (Gerber, « Le Cohelmec » 180)

Pour Drouet, par exemple, « un musicien indien se moque totalement de savoir si sa production sera conservée ou non. Ce qui compte, c'est ce qu'il a mis en marche à l'intérieur de lui-même » (in Gerber, « Le New Phonic Art » 201). Et Patrick Deletrez de témoigner : « L'improvisation n'est pas un truc que l'on peut juger vraiment. Pour ce qui me concerne, le jugement, par exemple, sur ce que je fais, m'importe vraiment peu. En fait, ça m'importe, mais ça ne m'impacte pas. Le bonheur de l'improvisation, c'est bien de faire. II est dans la pratique et bien peu dans le fait de plaire ou de déplaire » (octobre 2016). L'importance de l'improvisation tient alors à la praxis qu'elle nécessite de mettre en œuvre— « Je ne suis pas croyant, je suis pratiquant » (B. Lubat)—et, plus précisément, à ce que celle-ci réclame de celles et ceux qui s'y risquent. Sa singularité se voit ainsi liée à ce qu'elle est « un moyen conduisant à une évolution de la personnalité de chacun et de la vie quotidienne individuelle et collective » (Willener 207).

À Uzeste on joue à se grandir, à se développer, à s'autonomiser. Improviser, c'est jouer sa vie, ses désirs, ses connaissances, son ignorance. C'est une recherche intime où chacun va jouer avec ce qu'il devient et pas forcément toujours avec ce qu'il est. Le principe actif de ce à quoi nous jouons, ici, à Uzeste, est l'individuation. On cherche à se construire, à s'amuser d'être ; pas à se croire, plutôt à se pratiquer. Le jazz n'est pas seulement une forme de musique, c'est une tentative de libération, d'émancipation, de se libérer de l'esclavage, y compris de soi-même, c'est-à-dire se libérer de ce que l'on croît qu'on est. Et ça, ça n'a pas de prix, ça ne vaut rien d'ailleurs. (B. Lubat)

L'improvisation, c'est comme la liberté, c'est se souvenir de ce qui n'est pas encore arrivé. L'impro c'est d'abord du point de vue moral, l'impropre. C'est pas fini, c'est pas calibré, c'est pas contrôlé. C'est imprévu. L'impro c'est aussi penser, exprimer de la pensée par l'acte, le rythme, la parole, les sons. C'est une expression de l'individuation puisque chacun improvise nécessairement comme personne d'autre. Ceux qui y jouent, jouent comme personne. Jouer, c'est la seule possibilité d'humanité : on ne sait pas, mais on joue, ça ne nous empêche pas d'être. Quand on joue, il y a un faire qui te fait. L'individuation c'est un mouvement assez insaisissable, mais c'est ça la liberté. L'individuation et l'improvisation, là où elles sont le plus pointues c'est avec, contre, elles-mêmes et l'autre. Ce qui nous construit c'est cette relation. L'improvisation, c'est quand tu as les coulisses qui montent sur scène, c'est-à-dire ton inconscient et l'histoire, ton parcours. (B. Lubat)

\section{Individuation et Parrêsia}

Autrement dit, l'improvisation est une mise en Je(u) de soi permettant de se sortir des « cadences » de la musique et de la vie: "Nous sommes un laboratoire pour un art impopulaire, d'avant garde, expérimental, biodégradable. Nous sommes des joueurs, on joue à jouer, à se jouer. Nous sommes des renégats car nous ne voulons pas nous laisser simplifier. Nous nous improvisons nous-mêmes tel que nous l'entendons, si nous nous entendons » (B. Lubat). Lubat évoque souvent la nécessité d'une « musique à vivre » qui ne vaut rien, " désagréable à l'oseille », laquelle serait l'antithèse d'une musique « à vendre ». La mosique " sans papier » qu'il appelle de ses vœux et qui se pratique à Uzeste est, de facto, une musique vivante « qui n'a pas de prix » en ce qu'elle s'oppose à la « musique en boîte » des industries culturelles et au caractère fétiche de la musique (Adorno Le caractère) : « [Ici], on peut perdre son temps, on ne cherche pas à être beau ni puissant, ni juste, ni faux, ni vrai, ni laid, ni beau. C'est ça que j'appelle gratuit » (Lubat in Mittelberger et Zirn). Une musique est vivante ${ }^{19}$ parce qu'elle permet d' « expérimenter des inutilités radicales » (Lubat, « Intervention ») et mobilise le vif du sujet, ${ }^{20}$ c'est-à-dire ses capacités à devenir.

Improviser c'est être en situation critique, c'est se faire matière à un engagement. C'est s'inventer. C'est, comme le dit Glissant, le tremblement de la pensée ${ }^{21}$. C'est un jeu. Celui de jouer et de se jouer pour se déjouer, de devenir une œuvre à l'œuvre. II ne s'agit pas de se considérer comme une victime, un pingouin ou un dominant, mais comme quelqu'un en mouvement. Il s'agit de s'affranchir de ce que l'on croît de soi. Croire sur soi, c'est l'immobilité, penser soi, là c'est le mouvement. On est sans arrêt à l'œuvre. Et l'improvisation est cette espèce de charivari entre l'imaginaire, la danse et le rythme. C'est un puits sans fond et sans fin. L'improvisation est un art sans fin. II n'y a que des commencements. (B. Lubat, décembre 2016)

À quoi ça joue, à quoi ça sert ? Pour qui je joue ? Est-ce que je joue pour la révolution ou pour des bobos ? Est-ce que je joue pour Nuit debout, le CIP, des initiatives de lutte ? Je ne suis pas sûr que ce que je joue sur scène va insuffler des élans. Je n'ai pas la réponse, mais ce que je sais, c'est que moi ça me transforme de jouer sur scène, de me mettre dans ses états-là, de me mettre cette pression-là. Le message il est aussi dans l'état de transformation des "acteurs", des ouvriers de l'œuvre. Voir l'humain en action en train de se 
libérer de ses chaînes sur scène, c'est ça le plus important. Théâtre vivant ! T'es l'âtre de quoi ? Du bien calibré ou de la vie ? (J. Chao, avril 2016)

Je ne suis pas du tout musicienne. Pour moi, l'improvisation n'est pas une technique musicale, mais une posture de vie. J'ai toujours eu dans la vie ce côté : "c'est fini, on jette". Je n'ai pas de plans définis, pas de projets à long terme. Mon processus de vie est totalement improvisé. Si ça ne va pas à un endroit, je vais ailleurs. Je n'ai jamais rien calculé. L'improvisation est politique dans cette idée de ne pas faire ce qui est attendu, dans un cadre attendu qui sort le risque, l'imprévu et la poésie du faire. Improviser, c'est un engagement où tu laisses rien de toi en route, où tu emmènes tout de toi, même ton ombre. (L. Duthilleul, avril 2015)

L'improvisation est donc envisagée, à Uzeste, comme un « art de vivre libre » (Patlotch), une maïeutique permettant d'aller à la découverte d'un soi que l'on ne connaît pas nécessairement bien-i.e. de se différer-dans la mesure où celle-ci oblige à des réactions qui ne peuvent être réellement prévues : " c'est se lancer dans l'inconnu, l'advenir, l'àdeux-venir et à plusieurs »(B. Lubat, décembre 2016). Elle est une " expérience de quelque chose à vivre » (Quan Ninh 6), une relation poétique qu'il faut nécessairement éprouver pour en considérer la portée et par, conséquent, elle se présente aussi comme " une disposition à entretenir sa propre vitalité22 » (Quan Ninh 27). C'est en cela une éducation à soi et à l'autre, car il est bien clair que c'est par ce dernier que le Je(u) advient : "L'autre t'entraîne dans un autre de toi-même que tu ne connais pas encore, parce que l'autre est tellement l'autre que tu ne te suffis plus d'être ce que tu fus à la confrontation de l'autre » (B. Lubat, décembre 2016).

L'improvisateur est un Étranger Absolu de la musique, affirme le saxophoniste Michaël Attias. II peut parler toutes les langues et les hors-champs de la langue, bruits, cris, chuchotements, s'identifier aux bruissements du monde, se découvrir un devenir minéral, végétal, animal. Mais de langue propre, sauf par parti-pris idéologique, il n'en a aucune. Ou plutôt, c'est dans la mesure où il n'en a aucune qu'il m'intéresse, qu'il est moi, c'est-à-dire un autre. . . . II s'agit exactement de jouer à partir d'un Je devenu autre. Quand je joue, c'est le jeu qui me rend autre à moi-même, qui me dédouble entre celui qui fait et celui qui écoute, celui qui se concentre dans les profondeurs d'un silence intérieur et celui qui fait le bond. (2015)

À cet égard, l'improvisation est une pensée-pratique de l'errance, de l'exil intérieur ; non pas « l'éperdue pensée[pratique] de la dispersion mais celle de nos ralliements non prétendus d'avance, par quoi nous migrons des absolus de l'Être aux variations de la Relation, où se révèle l'être-commun-étant, l'indistinction de l'essence et de la substance, de la démesure et du mouvement » (Glissant, Philosophie 61). II s'agit en quelque sorte de laisser la place au précipice de chacun, à son ignorance, sa connaissance, ses peurs, ses désirs, ses habitudes, son imagination; de tout prendre et de ne rien épargner. Aussi, du côté de l'Estaminet, le néologisme "self'spliquer" ${ }^{23}$ » est souvent utilisé pour décrire ce travail d'avoir à « habiter pratiquement, poétiquement et politiquement sa peau, à traquer l'inexploré en soi, à jouer différemment et se jouer autrement, de s'émanciper et non de se perdre à jouer de l'instrument plutôt que sa vie » (B. Lubat, mars, 2017).

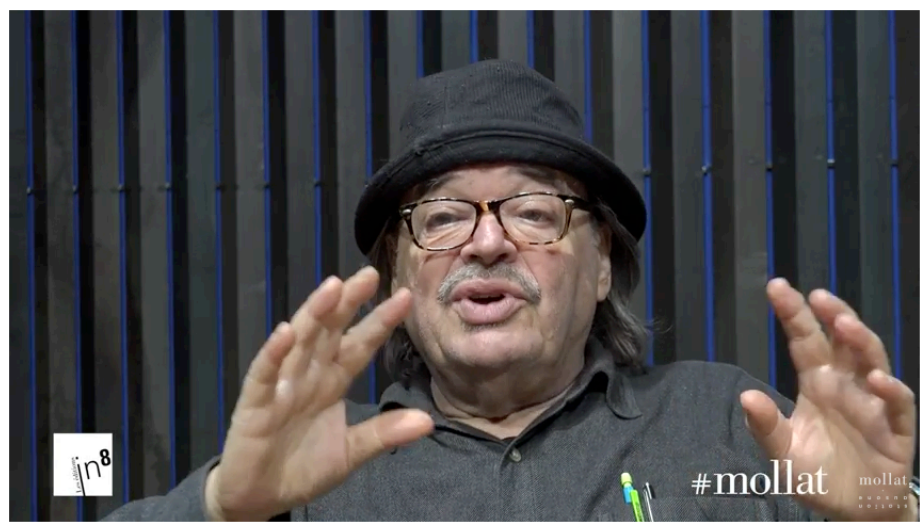

Extrait d'un entretien avec Bernard Lubat - Librairie Mollat https://youtu.be/_nXkKMtNrPg

L'improvisation peut être alors envisagée comme relevant d'une " esthéthique ${ }^{24}$ " de l'existence (Foucault, 2001a) visant l'auto-transformation, l'institution de soi, un exercice de soi sur soi, via l'autre, en opposition à des normes qui obligent à rester dans l'immobilité de ce qu'on est tel que la société nous a fait. Elle serait ainsi, comme une esthétique-éthique ${ }^{25}$ du refus, certes personnelle, " mais toujours adossé[e] à un souci du monde » (Macé, 2016 : 224). Par ailleurs, « Si rien n'est vrai, [mais que] tout est vivant » (Glissant, 2009 : 104), la vérité de soi ne se 
trouverait pas tant dans une immuabilité ontologique que dans la capacité à se mettre en Je(u) sur une scène collective en explorant des vies singulières, en créant de la différence, de l'altérité, un mouvement qui ne relève jamais de l'identique, mais toujours du Divers, de la variation, même la plus infime (métastabilité), c'est-à-dire ce par quoi la vie administrée peut se fissurer et ouvrir les voies à des imaginaires et des actions alternatifs ${ }^{26}:$ : une puissance morale et politique d'écartement » (Macé 225). Dans cette perspective, l'improvisation serait pour les " œuvriers » d'Uzeste " une matrice pratique de l'expérience de soi » (Foucault, Qu'est-ce que la critique ? 88) mêlant souci de soi-epimeleia heautou—souci des autres et souci du monde ${ }^{27}$; jonction que Lubat traduit de cette manière : «Si je devais me définir ? En fait je ne me définirais surtout pas : pas d'étiquette, pas d'identité révélée, que du devenir, de l'inconnu, de la transformation du souci en souci de la transformation ». Ici, le soi se définit non par une fixité, mais, a contrario, par un mouvement, une Relation : « ce n'est pas quelque chose de structuré, qui est donné au commencement [mais] certaines relations à soi-même » (Foucault, Qu'est-ce que la critique ? 117). Et cette relation à soi est nécessairement aussi une relation aux autres_Lubat évoque à cet égard une " esthétique des conjugaisons ». Sur scène (espace de représentation du politique uzestois), pour chacun des «œuvriers » en contexte d'exposition de soi, l'autre se compose des autres artistes, mais également du public, et pour ce dernier, l'autre, celui qui invite à se constituer un rapport à soi adéquat, ce sont les artistes.

Improviser pour moi, c'est parler pour soi et de soi. C'est trouver sa voie et trouver sa voix. C'est faire le point sur ce que l'on est : qu'est-ce que j'ai à apprendre, qu'est-ce que je sais ? Qu'est-ce que j'ai envie de lâcher avec ou sans contrôle ? Ce ne sont que des questions. Improviser, c'est à la fois prévoir et se laisser porter, c'est avoir confiance et douter. C'est se confronter à la réussite et à l'échec. L'improvisation, ça permet la rencontre. C'est une ouverture phénoménale à soi et aux autres en essayant d'être prêt à tout, c'est travailler sa capacité à rebondir en musique, mais dans la vie aussi évidemment. La musique, ça construit bien sûr des musiciens, mais aussi des artistes, des personnes pensantes, des humains entiers et complets. C'est une porte d'entrée sur le monde et c'est une façon d'être au monde avec d'autres. Personnellement, si j'ai pu me développer et arriver là où j'en suis en tant que musicien, mais aussi en tant que personne, c'est par le collectif. Parce que j'ai été couvé, aidé, mais aussi violenté. Le collectif ça crée de l'émulation, une bonne compétition, des tensions, des frictions, de la joie, de la colère. C'est presque une éducation de l'ego. Parfois tu as le droit d'être mégalo, prétentieux, d'autre fois tu te dois d'être très humble. Ça fluctue et la richesse est là, dans le fait de pouvoir vivre . . être effrayé, douter, être rassuré, avancer. (Jules Rousseau, bassiste de la Compagnie Lubat, avril 2016)

L'improvisation ça t'empêche de faire des trucs trop parfaits, et ça, c'est intéressant parce que ça fait tomber des barrières quant à ta capacité à affronter des obstacles depuis ce que tu es. Quand t'improvises tu joues ta vie, ta vie dans un contexte, une situation. Et la vie, c'est de l'improvisation. Moi, je suis dans un rapport constant à ça, à improviser pour trouver des solutions, parce que je n'ai pas le choix de faire autrement. Improviser c'est aussi affirmer une position dans un collectif, c'est-à-dire s'affirmer, mais dans le respect des autres et de la capacité commune de l'ensemble à construire quelque chose. Quand tu prends un solo, c'est toi qui conduis le bus, tu prends le relais, mais si tu conduis à un moment donné, c'est parce qu'il y en a un qui t'indique le chemin, l'autre qui te file de l'eau, etc. L'improvisation, c'est aussi une école de la collaboration. Tu n'es jamais tout seul. Bernard, par exemple, a cette qualité de laisser la place aux gens. Après libre au mec de la prendre ou pas et de la manière de la prendre s'il la prend. Ça te responsabilise grave. Mais dans l'improvisation, il y a cette idée de composer avec des personnalités qui peuvent être très différentes, mais il faut que tout le monde trouve sa place. (Thomas Boudé, guitariste de la Compagnie Lubat, avril 2016)

Le souci de soi se couple donc à une attention à l'autre et, de là, peut naître un respect des idiorrythmies (i.e. des rythmes personnels-Barthes) susceptibles d'organiser un faire commun et la possibilité d'un vivre ensemble différents. "L'improvisation mérite moins d'apparaître comme relevant d'une puissance d'expression que d'une puissance de constitution : non seulement elle favorise la production collective de singularités, mais elle promeut une solidarité active entre ces singularités » (Citton).

Se construire citoyen dans le collectif de travail, c'est se donner la capacité de grandir, de s'éduquer et de vivre à son rythme. Se laisser le temps de se construire, de se constituer. Le chantier de l'art c'est le chantier de la vie. Il y a un continuum car on transporte le même sac. L'un et l'autre sont les laboratoires de l'un et de l'autre. En même temps, on court tout le temps. Je ne suis pas pressé, mais en même temps très pressé d'en découdre. II y a là une contradiction, c'est évident. (J. Chao, avril 2016)

À l'instar d'Ernst Bloch (1977), pour qui la musique est une forme d'anticipation d'une vie qui ne serait plus mutilée, ${ }^{28}$ l'improvisation d'UZ se présente comme un aperçu de la puissance nécessaire à l'actualisation d'un futur espéré, lequel s'incarne au présent dans un choix d'existence. Elle est ce par quoi il devient possible de faire de sa vie une $œ u v r e$, non dans le sens d'une esthétisation stylistique de son existence, mais dans celui d'envisager le soi comme 
une structure éthique de personnalité à l'œuvre : non une subjectivité préalablement définie et vers laquelle il faudrait tendre ou qu'il faudrait retrouvée — fut-elle critique — mais plutôt un processus de subjectivation à conquérir en permanence sur le prescrit, l'attendu, le tolérable et leurs contradictions. « Le soi, c'est une œuvre d'art. C'est une œuvre d'art qu'on a à faire, et qu'on a en quelque sorte devant soi. . . . Le soi est donc une création, une création de soi-même : on se fait son propre soi » (Foucault, Qu'est-ce que la critique ?155).

Depuis notre place d'artistes, nous remettons les pendules à l'œuvre et tentons de maintenir un contrat d'engagement social pour un futur à vivre, à conquérir, à construire, à partager avec abnégation, courage, compétence, patience, et souvent avec souffrance devant certaines indifférences. Nous essayons de montrer qu'une vie n'est bien remplie que si l'on comprend qu'il faut tout une vie durant s'y apprendre, s'y cultiver, s'y interroger, s'y instruire, s'y critiquer, s'y inventer sans cesse de la naissance à la mort. C'est là notre responsabilité, notre mandat, que de montrer que d'ici d'en Sud-Gironde peuvent se façonner de vrais artistes citoyens concernés, impliqués, engagés qui respirent la liberté, la sensibilité, l'imagination, la responsabilité, ouverts sur le monde, sur l'autre, sur le différent(d) autant que sur le référent, dans le respect de l'héritage qui est ici le notre. (B. Lubat)

Lubat évoque ici une forme d'entreprise de « reddition de compte de soi-même » qui, comme le souligne Michel Foucault, nous ramène « au bios, à la vie, à l'existence et à la manière dont on mène son existence . . . : un mode d'existence qu'il s'agit d'examiner et d'éprouver tout au long de cette existence même » (" Intervention »148). L'existence (le bios) comme esthétique, c'est-à-dire la subjectivité comme objet pour une forme esthétique est, à Uzeste, un couplage particulièrement prégnant dans la mesure où il est à la fois la fin, mais aussi le moyen par lequel l'individuation prend corps. Autrement dit, l'improvisation relève d'une forme de parrêsia. Elle se conçoit comme un travail de constitution de soi, mais également comme une pratique de véridiction ${ }^{29}$ qui enjoint à prendre le risque de dire la vérité de soi, des situations et de pousser les autres à faire de même. Il s'agit alors de se construire au risque de la violence de la Relation et plus précisément de ses potentielles contradictions, c'est-à-dire du développement de propositions en tension, mais néanmoins dépendantes les unes des autres.

Ainsi, Serge Babkine (ingénieur du son de la série des disques Dialogiques_Labeluz) commente-t-il une prestation scénique de la Compagnie Lubat : « Votre musique est violente, elle violente les oreilles de ceux qui n'y entendent pas tout de suite ce pourquoi vous la faites ». Et Bernard Lubat et Fabrice Vieira de répondre : « [B. L. :] Oui tu as raison, ça me pose d'ailleurs question cette manière qu'on a de jouer. Ce soir on a été tout de suite dans le volume et la bagarre. Ça s'empoigne direct. C'est notre liberté, mais il y a comme une impossibilité d'exprimer cette liberté autrement que de cette manière. II reste donc une part de non liberté dans cette liberté. [F. V. :] Oui, mais je crois qu'on ne peut pas faire autrement. Quand on fait cet exercice, forcément on s'arrache. II y a un engagement et une intensité tout de suite qui s'expriment de cette manière. Si on exprime notre liberté, on l'exprime contre les réflexes que nous demande une société qui instaure des formes de domination terribles et s'y opposer c'est forcément violent. Ce qu'on exige est violent et ce qu'on se demande est violent ... (mars 2017)

On ne joue bien que contre ! C'est pour ça que l'improvisation est une situation difficile à supporter psychologiquement. Ça fait des dégâts car c'est la culture du doute total. C'est ça que je considère être mon existence. L'improvisation c'est quand d'un seul coup tu arrives à penser que c'est toi la partition. La partition c'est ta merde, ta vie : qu'est-ce que tu fais avec ça, avec tes goûts, tes dégoûts, tes désirs et ceux des autres. Et le public, face à ça, il n'est pas mis en face de ses goûts, mais d'une interrogation : "qui je suis moi ?". Cette suspension, cet heureux-bondi, ce swing qui ne tourne jamais deux fois pareil, c'est ce qui ouvre, ce qui "met en fuite" : fuir, ne plus être étanche de soi et se carapater, s'éloigner pour ne pas être repris par l'ordre qui est à tes trousses. (B. Lubat)

L'improvisation parrèsiastique est un courage « dont la forme minimale consiste en ceci que le parrèsiaste risque de défaire, de dénouer cette relation à l'autre qui a rendu possible précisément possible son discours » (Foucault, Le Courage 13). Cette vérité risquée ${ }^{30}$ de soi dans la situation est souvent définie par Lubat comme un " mentir vrai » (Aragon) ou une " mauvaise foi » : d'une part, se trouver dans l'obligation de rompre avec ses dispositions et de se délester du poids des déterminations (le mentir) pour, d'autre part, s'ajuster à l'autre par la Relation (la vérité du courage de l'affrontement) : "L'autre t'oblige à faire et dire derrière ce que l'on croît que l'on est, de ce que l'on a été éduqué. L'improvisation, c'est la possibilité de se trahir, comme on le dit d'un lapsus, c'est-à-dire la possibilité de se révéler à soi » (B. Lubat, décembre 2016).

La mauvaise foi est intéressante parce qu'elle est le mystère, le pas croyable. Elle n'a rien d'évident dans la mesure où elle doit être cultivée, mais elle permet le décalage permanent, la marge, le mouvement de la vie : ne jamais être là où on m'attend, être en capacité de stopper le fait de pouvoir croire que les choses doivent se passer plutôt d'une manière que d'une autre. L'intervalle, le rythme, l'improvisation, l'intranquillité, 
la mauvaise foi sont des éléments très proches les uns des autres parce qu'ils participent tous du jeu qui consiste à résister à se saisir pleinement de sa puissance d'agir et de penser pour défaire les « do-midances » et ne pas se laisser simplifier, jamais. (B. Lubat)

Parmi les techniques de soi ${ }^{31}$ qui ont vocation à permettre à l'individu dans le rapport qu'il entretient à soi-même et aux autres de se constituer en sujet autonome, il en est une qui, à Uzeste, s'avère particulièrement usitée : celle de l'obstacle. "L'obstacle comme lieu de passage » comme aimait à le rappeler André Benedetto, un passage ascétique qui « ouvre des continents invisibles, des continents intérieurs » (B. Lubat, décembre 2016). « [Uzeste], me paraissait être, par le lieu, son histoire, son espace, son temps, une somme de difficultés propres à approfondir le sujet » (Lubat in Lavaud 60).

Tout ce que l'on ne sait pas faire, il est utile de s'y confronter, notamment dans l'improvisation. Seul la cultivation d'un état de danger permet que se révèlent des ressources insoupçonnées. C'est mon instinct de conversation qui me pousse à ça. S'il m'arrivait un jour de croire à un genre, j'ai l'impression que je ne serais plus capable de progresser ! Je suis un "in-situ-actionniste". C'est extrêmement ludique et jouissif d'être, en face de l'autre, un "autre", que nous ne connaissons pas. J'aime, dans l'instant du jeu, que nous nous surprenions dans des régions qui nous surprennent, c'est-à-dire pas dans nos habitudes de la surprise. J'aime que nous nous disions ce que nous n'avions pas prévu de nous dire, et ça sur à peu près n'importe quoi. Cet endroit insituable, il est très précieux, parce que c'est précisément là que nous apprenons. C'est quand nous ne savons pas, quand notre ego n'est plus d'aucun secours, quand il y a simplement ce qui se passe, que nous nous perdons et que nous trouvons. (B. Lubat)

Rater, rater encore, rater mieux (Beckett) afin d'organiser une déprise d'avec un soi extro-déterminé puis d'initier une reprise de soi (Marx- « aller chercher notre liberté en nous-même »-B. Lubat, décembre 2016) que l'on espère moins dépendante des déterminations communes pesant sur le sujet. Willener évoque par exemple, s'agissant du groupe Faire, le principe d'un déconditionnement : "L'expression "se mettre à nu" devant l'événement sonore signale la nécessaire disponibilité-ne pas se barricader derrière le déjà su, connu, maîtrisé, s'exposer, en étant débarrassé de l'habitude » (218).

De même, Lubat affirme assez souvent qu'il s'est « mis minable » afin de décrire certaines de ses improvisations. Non que celles-ci aient été particulièrement ratées, mais plutôt parce qu'elles ont été des moments dans lesquels l'Amusicien d'UZ, en difficulté, n'a pourtant jamais flanché, n'a pas consenti à accepter ses faiblesses, ses manques, a résisté pour ne pas s'avouer vaincu par des situations qui, pour diverses raisons, ont pu être défavorables à l'expression de sa liberté et heurtantes s'agissant de sa dignité d'homme libre. "Ne jamais céder sur son désir » (Lacan, 1986) pourrait être une formulation équivalente à ce « mettre minable » lubatien. Désir de conjurer le pire et, pour ce faire, avoir le courage de s'engager, de s'opposer-celui d'essayer plus que de réussir-de ne pas se résigner, de prendre des risques, de déroger aux prescriptions et aux solutions " sur mesure ", de faire problème, de tenir bon plutôt que de faire $(d u)$ bien ou de faire correctement :

Il ne faut pas se préparer à improviser en faisant l'inventaire de prétendues munitions sans lesquelles on perdrait une bataille sinon la guerre d'une démonstration. II faut se préparer plutôt à tout laisser, à tout gaspiller à la première seconde, dans la première seconde, dans l'espace de cette première seconde. Quand il n'y a plus d'avoir à dépenser, reste la dépense de l'être. Et le rythme-ou bien ce qu'on pourrait être tenté de nommer l'esthétique—-serait peut-être fait des vitesses de cette dépense. (Quan Ninh 23)

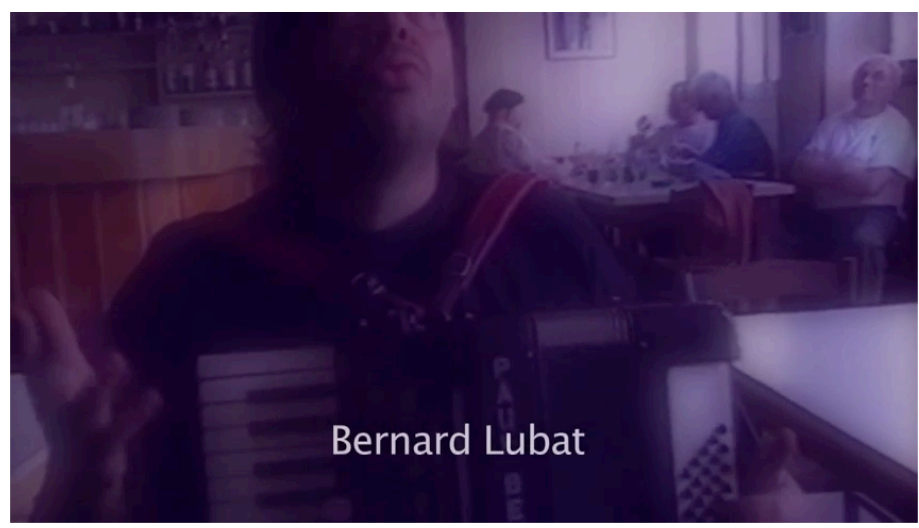

Performance musicale improbable de Bernard Lubat à L'Estaminet (Uzeste-France) https://youtu.be/IEW_Mf1zQ2C 
L'improvisation exprime alors, à l'instar du théâtre de Jean Genet commenté par Lucien Goldmann, « la dimension du possible et du dépassement » (93). Sa fonction critique tient ainsi, tout autant au travail de relativisation, voire de sape des ordres musicaux qu'elle permet, qu'à ce qu'elle montre en acte des alternatives et du désordre sensible qu'elle produit. Travail esthétique du négatif et critique pratique de l'existant, elle est, dans le même mouvement, une actualisation positive de possibles « non conformes ". L'improvisation est en ce sens un improbable qui ne cesse d'advenir, de surgir, qui fait événement. Elle se fait ouverture d'intervalles de dignité permettant de « tenter la possibilité d'une sculpture de soi » (Quan Ninh 37) et démonstration singulière de la possibilité d'alternatives :

À l'époque, s'il avait fallu réfléchir à ce que j'aimais faire précisément, ça m'aurait carrément bloquée. S'il avait fallu intellectualiser, rien ne serait sorti, c'est certain. Le mode de l'improvisation, c'était pour moi désinhibant, ça a été le transport qui m'a permis d'y aller, d'oser, de ne pas me poser trop de questions. Si j'avais pris conscience de ce que ça voulait dire que de partager la scène avec Édouard Glissant, Patrick Chamoiseau ou Miquel Barceló, évidemment, je ne l'aurais pas fait. À Uzeste, j'ai aussi appris à me mettre en résistance contre les choses qui me révoltent. Cette capacité à s'engager dans une bataille, c'est ça que l'on m'a transmis, d'être en capacité de construire des espaces de résistance qui sont d'ailleurs assez solitaires car chacun n'a pas la même cible et n'agit pas au même endroit. (Dalila Boitaud, comédienne, auteure, metteure en scène, avril 2016)

Mais l'improvisation est aussi la monstration de la compétence à produire ces alternatives (« tous capables ou avoir envie de l'être! Cette capacité qu'on a tous à être fragilement libre »—B. Lubat, mars 2017), ainsi que l'attestation de la nécessité d'avoir à s'engager ${ }^{32}$ pour arriver à ces alternatives. " Uzeste est un laboratoire dévoué à nos forces et surtout à nos faiblesses » (décembre 2016) affirme Lubat : laboratoire de production de processus de subjectivation, de structures de personnalité, d'étude de soi, de mise en confiance :

On se joue soi dans comme structure dans le flux des circonstance présentes. Se jouer soi. S'appréhender dans la complexité des mouvements qui fondent sa singularité. Dans le même geste, s'appréhender dans la singularité du moment présent, du lieu qui le fait apparaître, de sa géographie, de son architecture. Se jouer soi dans la simplicité de ses perceptions, cette simplicité qui s'élabore dans l'expérience, si lentement. Finalement, la structure qui se déploie, c'est-à-dire soi dans la relation avec ce qu'on perçoit, soi comme perception, c'est-à-dire soi qui disparaît. (Quan Ninh 63)

On se fait surprendre à rentrer dans l'improvisation. Elle a commencé à m'habiter assez tôt. Maintenant elle est en moi, je la cultive, mais c'est rentré tout petit. Bernard, avant d'être un maître en musique, c'est un maître de la "file-aux-autrie", de la transmission. II transmet ce goût de l'impro, de l'imprévu et d'être toujours en changement. C'est là où il est politique, dans sa manière de te montrer qu'il faut t'inventer, oser être ce que tu n'es pas encore. (J. Chao, avril 2016)

Aussi, plutôt que de jouer la réassurance d'une identité arrêtée de part et d'autre, Lubat propose de « se/nous planter »— " Je suis souvent pas d'accord demain avec mon accord d'aujourd'hui »—de s'/nous enraciner dans l'imperfection, l'imprévisible, l'imprédictible, de s'/nous affranchir douloureusement (car c'est toujours douloureux) des certitudes du résultat prévu. L'improvisation parrèsiastique est un cadre pour " se devenir et s'apprendre ». C'est une éducation à l'individuation intranquille, celle d'avoir à produire ses propres (impro)visions ; l'intranquillité ${ }^{33}$ d'avoir à détruire en soi ce qui nous rappelle à l'ordre, d'avoir à s'exiger un impossible présumé que l'on croît hors de portée et donc à s'engager : "L'improvisation, estime Lubat, ça fabrique des individus qui ne sont pas des couillonnés, mais des personnes qui se demandent, qui doutent, à commencer de ce qu'elles sont » (mars 2017). Si l'improvisation semble déterminer une expérience « de liberté d'action, de spontanéité, d'inconnu » (Portal in Levaillant 59), nombre de musiciens en rendent également compte comme l'expression d'une vérité provisoire de soi qui naîtrait du partage du chaos de la pratique. Lê Quan Ninh évoque ainsi ces « moments où dans la présence de tout ce que je perçois je m'aperçois d'un équilibre, d'une stabilité entre mon corps au travail et mon esprit en éveil, qui les mêlent et les confondent » (19). De même, le saxophoniste Ronnie Scott avance : " Quand l'inspiration, le duende, quel que soit le nom que vous lui donniez-une heureuse conjonction de conditions, d'événements et d'attitudes-est là, on se sent bien. On a l'impression que ce qu'on devrait être, on l'est vraiment. Ou quelque chose d'approchant » (Scott in Bailey 68). L'improvisation permettrait donc d'occasionner une euryhtmie des pratiques, des passions et des affects. Cet accord avec soi et avec les autres n'est toutefois qu'un moment d'un rapport bien plus heurté aux choses et qui caractérise le chemin qui nécessite toujours d'être parcouru pour y arriver. L'improvisation n'est donc pas seulement un comportement musical. S'y forger revient également à s'armer « pour la vie ${ }^{34}$ », comme en témoigne les travaux de Matthieu Saladin sur les groupes européens d'improvisation libre :

L'invention de soi représentait un mode d'être par lequel les musiciens s'interrogeaient sur leur rapport au musical, mais aussi plus largement sur la société dans laquelle ils vivaient et qui les avait en partie formés 
en tant qu'individus. Ils mettaient par là en question, à travers leur musique, ce qui les constituaient culturellement et sociohistoriquement. Les nouveaux modes de création sonores qu'ils expérimentaient collectivement avaient à la fois pour but tacite de créer de nouvelles relations entre les sons et d'engendrer de nouveaux rapports entre les musiciens eux-mêmes. (46)

Mais au-delà des musiciens eux-mêmes, le geste improvisé parrèsiastique en tant qu'il est une adresse à des publics peut avoir une portée politique plus étendue. "Que faire pour que d'autres en viennent aussi à faire [et] à être ", s'interroge Alfred Willener à propos de la portée sociale et politique de l'improvisation (236). Cette dernière est en effet, la plupart du temps, aussi une adresse à des publics dont il faut questionner la portée effective. Laure Duthilleul précise ainsi : "Le propre d'une création critique c'est évidemment d'essayer d'individuer les artistes, mais aussi les publics. C'est nettement plus exigeant que de se donner simplement en spectacle, c'est-à-dire se donner à voir en train éventuellement de s'individuer. Dans un cas, il y a participation au processus, dans l'autre, c'est simplement d'assister à ce processus " (octobre, 2016). II s'agit donc de faire art en essayant que les publics se saisissent du moment, non pas « à leur manière »—c'est-à-dire en conformité avec ce qu'ils croient être ou devoir sentir/pensermais précisément, depuis des appuis qui ne sont pas ceux culturellement appris et depuis lesquels ils ont l'habitude de réagir. Autrement dit, il s'agit de ne pas se conformer à l'expérience spectatorielle commune des publics, laquelle reste largement indexée à la rationalité marchande.

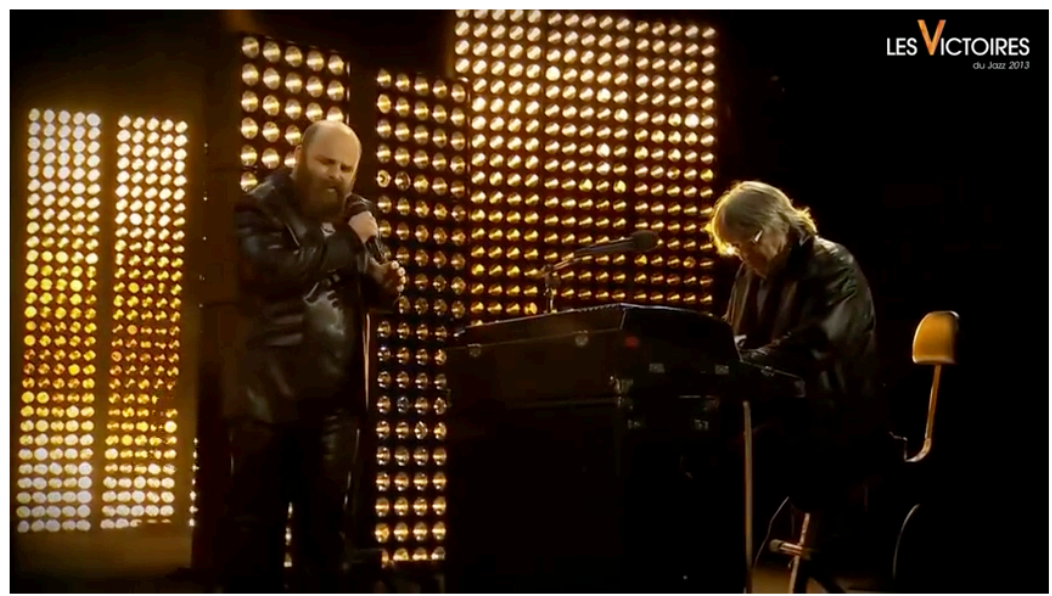

Les Victoires du jazz 2013 : Bernard Lubat et Thomas de Pourquery sur un texte de Pierre Desproges https://youtu.be/mlkLjzu8i1c

La chose est d'ailleurs d'autant plus importante si l'on considère, à l'instar de Theodor W. Adorno, que l'art ne résiste que par la forme - " et rien d'autre », ajoutait-il (Notes 289). Parier sur la pleine efficace d'un art critique nécessite donc de modifier les modalités de sa réception en essayant de désajuster structures institutionalisées de diffusion et dispositions incorporées de réception. Aussi s'agit-il d'inventer un art de la diffusion de l'art pour reprendre les propos de Lubat relevant, lui aussi, d'une forme de parrêsia en ce qu'il doit servir à « demander aux interlocuteurs de rendre compte d'eux-mêmes [et doit les conduire] effectivement à la découverte qu'ils sont bien obligés de reconnaître euxmêmes qu'ils ont à se soucier d'eux-mêmes » (Foucault, Le Courage 146) :

II faut à l'évidence chercher de nouvelles formes, de nouveaux comportements, un autre jeu avec la société, son époque, avec les lieux dans lesquels nous sommes amenés à manifester. Lors des premiers temps de la Compagnie, l'adresse au public s'effectuait sur le mode de l'interpellation : "Mais qu'est-ce que vous foutez là ? Vous n'avez que ça à foutre d'être public ?" Nous jouions la mauvaise fois, mais je ne supportais vraiment pas que les gens puissent par exemple rester assis, qu'ils se comportent juste en spectateurs. À Uzeste, quand nous avons voulu faire de même, évidemment, les gens ne sont pas revenus. Quand tu rentres dans le chou du public, forcément il s'en énerve, mais c'est une bonne chose car nous n'avons pas vocation à répondre à ce qu'ils croient être leurs attentes. Ça nous a également permis, nous, de ne pas nous laisser avoir parce que nous croyions devoir faire. Ça nous a permis d'être cultivés par le doute et de ne pas nous asseoir confortablement dans quelque chose que nous imaginions savoir faire, ni défaire. (B. Lubat)

Commentant une tournée effectuée dans le cadre de l'Europa Jazz Festival 2016, Louis Lubat (batteur de la Compagnie Lubat) témoigne : "Le public est venu nous dire à chaque fois : "mais vous êtes libres !". Des publics très différents pourtant. On montait sur scène et à chaque instant on commençait, c'était très ouvert, 
très libre. C'était en dehors des esthétiques. On ne savait pas vraiment où on était. Ça n'avait ni queue ni tête, c'était sans style. Là, on est allé plus loin que d'habitude, sans la pression de devoir jouer comme ceci ou cela en se demandant "de quoi j'ai l'air ?". La peur du ratage a disparu . . . Et ça, les gens, tu sens que ça leur plaît. Ils voient bien qu'on monte sur scène à poils et qu'on joue notre vie devant eux » (avril 2016).

Difficile à déconstruire, la rupture conventionnelle entre la scène et la salle, les artistes et leurs public, le produire et l'appropriation entraîne un différentiel d'expérience qui rend plus incertaine la transitivité du processus et plus évidente le partage de son résultat sonore ${ }^{35}$ ou plus largement artistique. Toutefois, ce dernier n'est pas sans puissance politique comme en témoigne cet uzestien déçu mais non résigné :

Tout de même, j'ai l'impression qu'il y a une contradiction à Uzeste, entre une puissance et une qualité artistiques qui continuent de s'actualiser, et une velléité politique qui "s'archaïse", bien qu'elle ait été, à un moment donné, elle aussi sans doute d'une très grande pertinence. Mais là où c'est complexe, c'est que ce moment passé brille encore, et a toujours un effet sur le réel. Toujours est-il que c'est cette qualité artistique présente qui me permet de croire à cette utopie que je ne vois pas, mais dont je devine les restes, et donc aussi la potentialité future. (février 2017)

Une mélancolie du peut-être, où l'être se définit d'abord par ses possibilités et par l'aléatoire de son mouvement, donc par le pari, le risque. Une mélancolie qui n'ignore pas le douloureux divorce entre le probable et le possible, mais s'attache à le surmonter, malgré tout, envers et contre tout. Dans la ferme certitude de l'incertitude, elle affronte le poids du doute, sans pour autant s'en défaire.

Daniel Bensaïd, « Engagement et neutralité du savoir »

L'improvisation d'UZ est une manière, nous l'avons vu, de se « constituer en tant que sujet éthique » (Foucault, Qu'est-ce que la critique ? 115). Parrêsia tenant donc, en premier lieu, à la sphère de l'éthique personnelle, elle n'est toutefois pas étrangère à une pratique politique désindividualisée ${ }^{36}$ c'est-à-dire à l'organisation d'un commun en partant du « chaos absolu de différences » (Arendt, Qu'est-ce que la politique ? 168). À leur manière, les « œuvriers d'ici d'en bas » en tant qu'« êtres différents » et cultivant leurs singularités d'artistes et de citoyens développent un « style d'existence » qui est un témoignage par la vie manifestant « directement, par sa forme visible, par sa pratique constante et son existence immédiate, la possibilité concrète et la valeur évidente d'une autre vie, une autre vie qui est la vraie vie » (Foucault, Le Courage 170). Lubat et Cie forment ainsi une communauté qui, de par l'existence même qui est la sienne, tend à donner témoignage de ce qu'est l'art de l'improvisation dans sa vérité, et porte, par là, une charge politique spécifique (biopolitique) : « l'art est capable de donner à l'existence une forme en rupture avec toute autre, une forme qui est celle de la vraie vie » (Foucault, Le Courage 172). L'improvisation parrèsiastique établit bien au réel « un rapport qui n'est plus de l'ordre de l'ornementation, de l'ordre de l'imitation, mais qui est de l'ordre de la mise à nu, du démasquage, du décapage, de l'excavation, de la réduction violente à l'élément de l'existence » (Foucault, Le Courage 173): "L'improvisation, c'est dire merde à la culture de ce que tu as reçu du social et du politique. L'improvisation c'est la nécessité de rompre. Si j'allais jusqu'au bout, j'insulterai les gens, je leur dirais "rentrez chez vous", mais je ne suis pas assez modeste pour faire ça. Ça m'est arrivé plusieurs fois dans des concerts d'improviser ma fuite. Se tirer ! » (B. Lubat, avril 2016).

L'improvisation est, en cela, une pratique de liberté qui (se) donne à voir et à entendre, au travers des propositions artistiques dont elle est au principe. Elle se fait « mise en scène » d'un politique (une politique de la scène et de la vie) et se présente comme un modèle d'une pratique cumulée de liberté qui produit de la différence, transforme l'individu, sans que le fruit de cette transformation ne soit prévisible à l'avance et sans qu'il soit à même de produire un processus plus large de libération: "L'improvisation, ça promet de cultiver des problèmes. Ce que nous faisons à Uzeste, c'est ça, on cultive les problèmes mais on voit bien que la récolte est maigre. Ça n'a pas pris à hauteur de nos espérances pour le moment. C'est du temps long » (B. Lubat). De facto, l'art critique d'UZ se heurte à la difficulté d'avoir à transformer l'improvisation parrèsiastique en une modalité/nodalité organisée dans un rapport de forces déterminé susceptible de peser sur le cours des vies uzestoises. Or sa nature même, installant le sujet « dans le mitan instable de toute chose » (Ménil 241), rend cet effort difficile. Non que celle-ci ne soit pas politique-elle l'estmais parce que la liberté critique qu'elle promeut tient singulièrement d'un détachement des formes organisées. II s'agit en effet de ne jamais céder complètement aux formalismes—fussent-ils critiques—qui sont toujours l'expression d'un ordre, de normes, de procédures qui figent pour partie les manières d'être, d'agir et de penser par l'énoncé d'attendus qui font règles et auxquels il serait utile, intéressant, voire nécessaire de se plier. On se plaît alors à imaginer un politique singulier qui s'improviserait :

L'improvisation a ça de jouissif qu'elle est une transgression des règles, mais elle n'ignore pas les règles. Le politique ça devrait être ça : établir des règles pour cadrer le commun, mais aussi être en mesure 
d'improviser sur ces règles, de mettre ces règles en marche. Et c'est par l'improvisation que la chose devient possible. La musique, c'est un endroit où ce type de choses se joue : un rythme commence, il est une proposition, tu peux aller avec, tu peux aller contre, tu peux transgresser la règle, mais il faut que la musique continue à se tenir. Un politique idéal, ce serait la même chose. (F. Vieira, avril 2016)

À Uzeste, on rêve l'irruption de sujets politiques imprévisibles, on travaille même à les faire émerger en « dissipant les "passions tristes" (Spinoza), [en composant] des rapports neufs avec le monde, de joie, de jeu, d'émulation, d'amour " (Moussaron 108), en portant haut les principes de la Relation et de ses incertitudes, en accordant "l'imaginaire de l'imprévisible avec les nécessités du faire et de l'agir » (Glissant, Philosophie 108), en tentant de penser ce qui pourrait être décrit comme une pluriversalisme, c'est-à-dire l'existence « d'un monde où de nombreux mondes aient leur place » (Garcia 115) et nous garderaient « d'être persuadés d'une essence ou d'être raidis dans des exclusives » (Glissant, Traité 26). Cette éthique poïélitique qui porte la nécessité de formes de vie composites est assurément un pari mélancolique (Bensaïd) qui s'incarne dans un désir de transformation de soi, des autres et du monde qui passe par des conflits à proprement parler politiques. Résolu, convaincu, il n'en reste pas moins traversé par une intranquillité fondamentale qui prend pleine conscience de la fragilité des personnes, des luttes qu'ils mènent malgré elle et de l'héritage des vaincus. L'improvisation parrèsiastique peut paraître éloignée, à première ouïe, de cet élan mélancolique ; elle en est pourtant l'expression singulière dans l'ordre de l'art et de la Relation, puisqu'elle fait fond sur un imprévisible et parie sur des potentialités libératoires qu'il resterait à mettre " à l'œuvre ", c'est-à-dire à faire vivre en chaque individu, puis en une praxis collective. Sans doute s'agit-il là d'un optimisme de la volonté, mais celui-ci n'a, pour autant, rien du décret arbitraire ou de l'avant-garde morale. Il est l'espoir raisonné et concret maintenant la fragile luminescence des « LUZioles » résistantes « d'ici d'en bas » et la possibilité d'un art politique « capables de mettre en œuvre un épanouissement humain qui s'inscrit dans l'horizontale plénitude du vivant » (Breleur et al. 12).

II contribue, à sa manière-i.e. modestement-à entretenir une certaine « folie d'être » (de Certeau 222), à valoriser le risque d'exister et à maintenir possible un avenir commun en faisant « surgir la différence comme pouvoir de révision des normes, . . . impliqu[ant] que les liens de l'individuel et du collectif ne soient pas scellés par un impératif de conservation ou de répétition sociale, mais donnent lieu au contraire à des renouvellements minoritaires » (Le Blanc 64). C'est bien là une (impro)vision politique qui met au cœur de son principium le fait que chaque individu se révèle à soi et aux autres (se singularise) comme sujet politique [et comme artiste d'exécution initiateur d'actions " concertées " (Arendt, La Crise)] par la manière qu'il a d'agir avec, contre et devant les autres, sur scène, dans les coulisses et dans la vie. C'est dans et par l'action que née l'ouverture à l'altérité et au Tout-monde ${ }^{37}$ glissantiendont Alain Ménil précise qu'il est « moins l'objet d'une expérience que l'énoncé d'un projet (575)—qui, pour se faire espace de concitoyenneté, doit passer par la construction, en chacun de ses archipels, d'espaces d'apparition et d'action. Uzeste est sans doute un de ces îlots (un lieu) et montre que pour passer de l'agir-ensemble au vivreensemble, il faut à la fois faire place aux identités spécifiques tout en se donnant les moyens de les « dépasser » sans les araser, par l'invention d'un monde commun solidarisant les différentes vivacités critiques du réel.

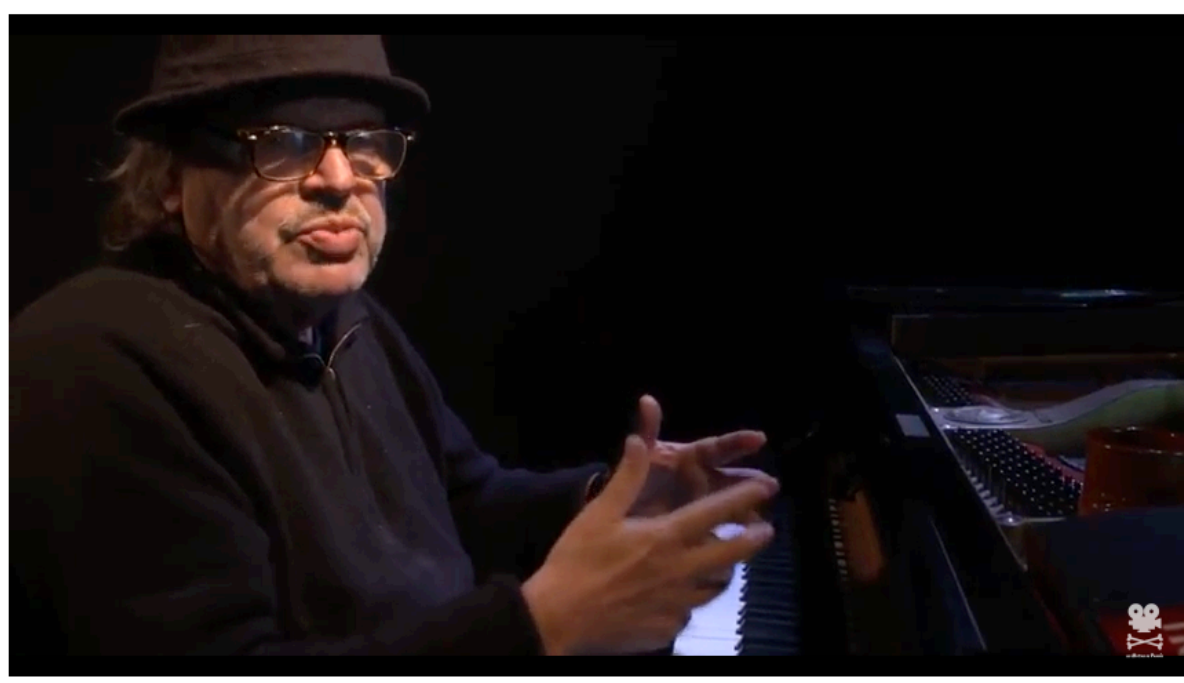

Bernard Lubat dans le doute à L'Estaminet (Uzeste-France) - Les Mutins de Pangée https://youtu.be/P6Z6mM-HxYU 


\section{Notes}

${ }^{1}$ Ces collectifs signent ce que d'aucuns nomment la « deuxième vague » du free français : Free Jazz Workshop de Lyon (1967), Full Moon Ensemble (1967), Le Cohelmec (1968), X-Tet, Celestrial Communication Orchestra (1969), Dharma, Perception, New Tone Experience (1970), Machi Oul (1971), Intercommunal Free Dance Music Orchestra (1972), Confluence (1974), Moravagine (1975), Rhizome (1976), etc.

2 Laquelle peut également permettre une « juste connaissance du réel », mais sans jamais s'y résumer complétement. Sur ce point, cf. (Brohm). Bertolt Brecht proposait que le théâtre rende étrange ce qui est familier (368-369).

${ }^{3}$ Barre Phillips, contrebassiste de free music, parle pour sa part d'improvisation non-référentielle.

${ }^{4}$ À l'instar d'André Breton, Léon Trotsky et Diego Rivera qui, dans leur manifeste Pour un art révolutionnaire indépendant exigeaient « toute licence en art ».

${ }^{5}$ Bernard Lubat ajoute : il faut « arriver à entendre le mot "jazz" pas seulement dans son aspect étiquette esthétique, ou période du jazz, puisque ça c'est le commerce qui fabrique le goût, mais entendre le jazz comme processus philosophique. C'est-à-dire que c'est archaïque, ça n'a pas d'âge. Le jazz existait même avant d'avoir été appelé jazz, un peu comme le torrent qui dévale depuis des siècles le Pas de Roland » (Lubat in Rivière 42).

${ }^{6}$ Hommage de Charles Mingus à Lester Young (Mingus Ah Um, Colombia Records, 1959).

${ }^{7}$ Pour Glissant, la répétition est « un mode avoué de la connaissance. Reprendre sans répit ce que depuis toujours vous avez dit. Consentir à l'élan infinitésimal, à l'ajout, inaperçu, peut-être, qui dans votre savoir s'obstinent » (Poétique 57).

${ }^{8}$ Sauf mention contraire, les extraits d'entretien avec Bernard Lubat sont tirés de Les UZ-topies de Bernard Lubat (Granjon).

9 À Uzeste est souvent utilisé le terme de « mosique » pour désigner un mélange de musique, de bruits et de paroles. Bernard Lubat évoque parfois sa Compagnie en la qualifiant de collectif «transartistique de divagation ».

${ }^{10}$ R. Keith Sawyer estime, par exemple, que l'improvisation conversationnelle se compose d'ajouts incrémentaux et créatifs qu'il appelle des offres et qui suscitent des réponses. II précise : "Une offre n'entre pas dans le cadre tant qu'elle n'a pas été acceptée par les coparticipants à la représentation. Ils ont collectivement la possibilité d'accepter l'offre (en travaillant à partir d'elle et en construisant sur sa base), de rejeter l'offre (en continuant la performance comme si elle ne s'était jamais produite), ou de l'accepter partiellement (en sélectionnant un aspect à partir duquel la développer, et en ignorant le reste). Cette décision évaluative est un travail de groupe, et le dialogue peut se poursuivre quelques temps avant que n'apparaisse clairement ce qui a été ou non accepté » (57).

11 Titre d'un spectacle de Bernard Lubat désignant ce dernier.

12 Jean-Pierre Drouet est un percussionniste virtuose qui s'est illustré dans divers domaines musicaux : classique, contemporain, improvisation libre, variétés et aussi le spectacle vivant : théâtre, danse, art équestre, etc. II a participé à plusieurs reprises aux hestejadas de las arts uzestoises.

${ }^{13}$ L'art est passé par des siècles d'oralité, manière de transmettre avec le moins de préalable possible, en faisant confiance à l'apprenant : la sensibilité et la mémoire interne davantage que la raison et les mémoires externes ; par le faire-pratique plus que par le faire-théorique.

${ }^{14}$ Pour Michel de Certeau, « la création est une prolifération disséminée. Elle pullule. . . . La création est périssable. Elle passe car elle est un acte. . . . L'œuvre périt donc avec le présent qu'elle symbolise . . . , elle vise à s'évanouir dans ce qu'elle rend possible. De là deux aspects importants de la culture. D'une part, prise dans l'éphémère alliage collectif dont elle cristallise un moment la possibilité, vouée à disparaître avec lui, l'expression culturelle relève à la 
fois de l'instant qu'elle marque, et de la mort où elle retourne. Elle représente un risque qui ne saurait être arrêté en l'un des signes, tel un oiseau métamorphosé en pierre » (de Certeau 214 et 215).

${ }^{15}$ Les improvisateurs sont parfois « décrits comme des acteurs suffisamment disponibles à la situation présente pour y puiser des ressources d'action et réagir sur le vif, par exemple à des réactions d'un auditeur, ou à des imprévus menaçant de troubler la situation d'écoute. La créativité réside ainsi moins dans la libération par rapport à un langage musical (thèse commune dans le champ de l'improvisation libre) que dans une forme de "prévoyance" (Bourdieu, 2000), une disposition à agir qui se traduit par une attention de chaque instant aux divers appuis de la situation » (Bachir-Loopuyt et al. 8).

${ }^{16}$ Chez Foucault, les termes sont inversés, la liberté étant une pratique individuelle, tandis que la libération, un processus plus ample (cf. infra).

${ }^{17}$ Stephanie Hill estime pour sa part qu'il existe fondamentalement deux aspects aux « moments d'improvisation » : l'un relationnel qui relève de la rencontre pratique des musiciens et l'autre représentationnel, qui serait le produit de cette rencontre.

${ }^{18}$ La similarité est évidente avec l'approche glissantienne de la créolisation, laquelle « consiste moins à louer le résultat $[\ldots]$ qu'à cerner très subtilement la spécificité des processus actifs et créateurs de la créolisation ... » (Ménil 618).

19 « Est-ce que tout cela est vivant ou est-ce que tout cela est mort ? » (Steve Lacy in Bailey 72) ; « Beaucoup trop de spectacles ne sont que des inventaires d'objets déjà morts » (Quan Ninh 40).

20 «L'improvisation n'est pas une manière d'inventer la musique ou de la jouer. Elle est cette invention elle-même. Elle est cette invention dont la source fondamentale se trouve au cœur du musicien, à la source de son être et de sa vie la plus intime. Elle est comme son cœur qui bat. Elle est comme ce rythme de la vie qui nous habite et que nous sommes, qui nous fait vivre et qui nous définit » (Arcens 44).

21 « La pensée du tremblement éclate partout, avec les musiques et les formes suggérées par les peuples. Elle nous préserve des pensées de système et des systèmes de pensée. Elle ne suppose pas la peur ou l'irrésolu, elle s'étend infiniment comme un oiseau innumérable, les ailes semées du sel noir de la terre. Elle nous unit dans l'absolue diversité, en un tourbillon de rencontres. Elle est l'Utopie qui jamais ne se fixe et qui ouvre demain : comme un soleil ou un fruit partagé » (Glissant, La Cohée 33).

${ }^{22}$ Sous cet aspect, l'improvisation est créolisation, en ce qu'elle a à voir « avec ce qui, dans l'expérience même du vivre dès lors qu'il s'agit d'un "vivre en commun", témoigne de l'aptitude de la vie "commune" à se maintenir, à se poursuivre, à se tenir prête ou à faire face » (Ménil 640).

${ }^{23}$ I.e. le fait d'avoir à se « cultiver » : une culture de soi (selbstbildung).

${ }^{24}$ Ce néologisme condense l'idée foucaldienne de « L'art de l'existence et le discours vrai, la relation entre l'existence belle et la vraie vie, la vie dans la vérité, la vie pour la vérité ... L'émergence de la vraie vie dans le principe et la forme du dire-vrai (dire vrai aux autres, à soi-même, sur soi-même et dire vrai sur les autres), vraie vie et jeu du dire-vrai » (Foucault, Le Courage 150-151).

${ }^{25}$ « Qu'est-ce que l'éthique ? C'est, je crois, la façon dont les sujets se constituent eux-mêmes en tant que sujets moraux dans leur activité et leur action, etc. Le problème n'est donc pas de développer le soi, mais de définir quel type de relation à vous-même est capable de vous constituer en tant que sujet éthique. II ne s'agit pas du développement du soi, mais du problème de la constitution de soi » (Foucault, Qu'est-ce que la critique ? 114).

${ }^{26}$ " C'est dire qu'on ne rend pas compte d'une œuvre quand on exhume les codes auxquels à son insu elle obéit. II s'agit là seulement des structures d'où elle émerge en les signifiant encore. Mais elle existe précisément par l'interstice ou la marge qu'elle y ouvre, sans cesser d'être sous la dépendance de lois sociales, psychologiques, linguistiques. Elle insinue un surcroît, un excès, et donc aussi une faille dans les systèmes d'où elle reçoit son support et ses conditions de possibilité. Un jour se produit dans un espace construit. Il en déplace l'équilibre, sans pour autant lui échapper. Là, il y a du jeu. C'est la zébrure d'une bouffonnerie : un divertissement, une transgression, une traversée "métaphorique", un passage d'un ordre à un autre, un oubli fugitif à l'intérieur des grandes orthodoxie 
de la mémoire. Tous ces mouvements sont relatifs à des organisations et à des continuités. Mais ils y introduisent la modeste prolifération d'une créativité. Ce grignotement de l'inventivité dans les marges des textes légaux dit le plus fragile et le plus essentiel de l'action humaine. L'innombrable variante qui pullule, telle une moisissure, dans les interstices des ordres micro- et macro-physiques, c'est notre culture » (de Certeau 215-216).

${ }^{27}$ Elle est, en cela, aussi une poétique. Glissant définit celle-ci comme « une manière de se concevoir, de concevoir son rapport à soi-même et à l'autre et de l'exprimer » (L'imaginaire 44).

${ }^{28}$ D'une manière plus générale, on peut considérer avec Jean-Marc Lachaud que les artistes transgressifs-i.e. dérogeant à une série d'attendus quant au style, au rapport au public, etc.- - sont ceux qui « critiquent et dénoncent la réalité existante, tout en fouillant, par la pratique indocile de leur art conçue comme une utopie concrète, d'inimaginables horizons à-venir, possibles-impossibles » (18).

29 " C'est l'ouverture qui fait qu'on dit, qu'on dit ce qu'on a à dire, qu'on dit ce qu'on a envie de dire, qu'on dit ce qu'on pense devoir dire parce que c'est nécessaire, parce que c'est utile, parce que c'est vrai » (Foucault, L'herméneutique 348).

30 « Dans la parrêsia, quel que soit le caractère habituel, familier, quasi institutionnalisé de la situation où elle s'effectue, ce qui fait la parrêsia, c'est que l'introduction, l'irruption du discours vrai détermine une situation ouverte, ou plutôt ouvre la situation et rend possible un certain nombre d'effets qui précisément ne sont pas connus. La parrêsia ne produit pas un effet codé, elle ouvre un risque indéterminé. Et ce risque indéterminé, il est évidemment fonction des éléments de la situation » (Foucault, Le gouvernement 60).

31 "Les "techniques de soi", c'est-à-dire les procédures, comme il en existe sans doute dans toute civilisation, qui sont proposées ou prescrites aux individus pour fixer leur identité, la maintenir ou la transformer en fonction d'un certain nombre de fins, et cela grâce à des rapports de maîtrise de soi sur soi ou de connaissance de soi par soi » (Foucault, Dits 1032).

32 Steve Lacy ne dit pas autre chose : « Je suis attiré par l'improvisation en raison d'une qualité que j'apprécie : la fraîcheur, que l'on ne peut obtenir que par l'improvisation et absolument pas par la musique écrite. Cela à avoir avec cette idée de "fil du rasoir" . . ., avec l'idée d'être toujours au bord du gouffre et prêt à y sauter. Quand on s'y lance, on a beau avoir des années de préparation, une certaine sensibilité et une certaine expérience, cela n'en reste pas moins un saut dans l'inconnu. On peut, en effectuant ce saut, découvrir des choses intéressantes qu'on ne parviendrait pas, je crois, à découvrir autrement » (Lacy in Bailey 73).

${ }^{33}$ L'intranquillité fait bien évidemment référence à l'œuvre de Fernando Pessoa. Celle-ci n'a pas vocation à changer la vie, mais d'abord à changer sa vie : « tout convertir en notre substance la plus intime » (Pessoa 39). II s'agit d'un acte créateur critique, mais autocentré, a priori sans adresse autre qu'à soi-même, dont la portée serait donc essentiellement individuelle. Et en même temps, son dégagement du monde (évasion) se couple à un engagement d'une grande force (conquête) dans l'écriture dont il pense qu'elle peut avoir valeur d'adresse : aller au bout d'une quête de soi, ne pas déroger, ne pas concéder, ne pas s'accommoder, " persister tenacement » et faire de l'existence « la veille, ou les environs, de quelque chose d'autre » (Pessoa 115). II y a, à l'évidence quelque chose d'uzestois dans cette dialectique dégagement/engagement. De même, si la geste pessoenne a pour principe de rêver la vie pour vivre le rêve, il est, " d'ici d'en bas ", entendu qu'il faille vivre le jeu pour jouer la vie. Dans les deux cas, l'art ou l'improvisation ont vocation à libérer le sujet en lui offrant la possibilité de se déterminer par soi-même à penser-ce qui correspond, selon l'auteur portugais, à l'exigence de la nature de l'homme et à sa nécessité intérieure-mais aussi à agir : vivre pour se dé-mentir. Pour Pessoa, le poète est un fingidor, sa faculté maîtresse n'est pas le don de « sentir », mais celui d'imaginer et de feindre (Bréchon 237).

34 « La vie, c'est évidemment une improvisation. Rien n'est marqué, rien n'est défini. II ne faut surtout pas penser que c'est fatal la vie. C'est là, pour moi, que la vie est une improvisation mais on ne le sait pas au départ » (André Minvielle-ex-membre de la Compagnie Lubat—in Lallement et al.).

${ }^{35}$ La chose est rendue d'autant plus difficile quand on passe de la scène vivante à la trace de l'action sonore. Sur disque, le mouvement de production de sons s'amenuise en effet considérablement au profit de son aboutissement, renforçant ainsi la charge esthétique de l'improvisation aux dépens de son caractère processuel. Seuls les plus connaisseurs peuvent facilement reconstituer les modalités pratiques de fabrication du text et, par là, ses aspects les plus politiques. Si la forme n'est évidemment pas détachée du fond, elle encode ce dernier au point d'en rendre 
l'accès plus âpre et difficile, comme en témoigne cet habitué de l'Estaminet : «Les concerts de free, là où ça part en sucette comme Lubat sait très bien le faire, j'adore ça. II se passe vraiment quelque chose. Sur disque, je trouve ça nettement moins intéressant. Ça perd en intensité, ça devient plus du bruit, alors que quand tu es là pour le vivre, il se dégage tout autre chose de plus charnelle. En concert, ça me touche dix fois plus, alors que sur CD ça va vite m'emmerder » (août 2016).

${ }^{36}$ Glissant estime « que le projet de l'existence individuelle suit généralement le cours de la recherche d'une existence collective » (Glissant et Leupin 37).

${ }^{37}$ Étienne Tassin souligne que « toute politique authentique est une cosmopolitique. Toute véritable politique est une politique du monde, du "cosmos", une cosmopolitique, non pas au sens d'une mondialisation des rapports humains ou de l'institution d'une société universelle (ce qui obéirait encore au principe communautaire), mais au sens où les républiques sont les lieux d'un monde commun possible (et ne sont pas de communautés identificatoires). . . . Tel serait alors le sens authentique d'une citoyenneté du monde : être citoyen du monde n'est pas appartenir à une communauté mondiale-à une humanité qui serait tout entière apatride de vouloir être à elle seule une seule communauté - c'est réfléchir sa propre inscription dans, et sa propre appartenance à, une ou des communautés finies_mais dans la perspective du monde commun »(147 et 149).

\section{Bibliographie}

Adorno, Theodor W. Le caractère fétiche dans la musique. Allia, 2001.

---. Notes sur la littérature. Flammarion, 1998.

---. Théorie esthétique. Klincksiek, 1974.

Arcens, Michel. John Coltrane. La musique sans raison. Alter ego éditions, 2012.

Arendt, Hannah. La Crise de la culture. Gallimard, 1972.

---. Qu'est-ce que la politique ? Seuil, 2014.

Attias, Michaël. « Réflexions personnelles sur les liens et les ruptures de lien entre l'improvisation musicale, le langage et la notion d'identité », Critical Studies in Improvisation, vol. 10, no. 2, 2015. doi: http://dx.doi.org/10.21083/csieci.v10i2.3344

Bachir-Loopuyt, Talia, et al., « Éditorial ». Tracés, no. 18, 2010, pp. 5-20.

Badiou, Alain. Pornographie du temps présent. Fayard/France Culture, 2013.

Bailey, Derek. L'improvisation. Sa nature et sa pratique dans la musique. Outre Mesure, 1999.

Barthes, Roland. Comment vivre ensemble. Simulation romanesque de quelques espaces quotidiens. Seuil, 2002.

Bataille, Georges. « L’Art, exercise de la cruauté. » Médecines de France, no. 4, juin 1949.

Bensaïd, Daniel. Le Pari mélancolique. Fayard, 1997.

Bloch, Ernst. L'esprit de l'utopie. Gallimard, 1977.

Bonnerave, Jocelyn. « Improviser ensemble. De l'interaction à l'écologie sonore ». Tracés, no.18, 2010, pp. 87-103.

Bréchon, Robert. L'innombrable. Un tombeau pour Fernando Pessoa. Christian Bourgois éditeur, 2001.

Brecht, Bertolt. Écrits sur le théâtre. Gallimard, 2000. 
Breleur, Ernest et al., Manifeste pour les « produits » de haute nécessité. Galaade éditions, 2009.

Brohm, Jean-Marie. « Les marxismes et les arts ». Prétentaine, no.6, 1976, pp. 171-185.

Canonne, Clément, et Pierre Saint-Germier. « De la philosophie de l'action à l'écoute musical. Entretien avec Jerrold Levinson ». Tracés, no.18, 2010, pp. 211-221.

Chemillier (Marc), « Le jazz, l'Afrique et la créolisation. Entretien avec Bernard Lubat », 2008. http://ehess.modelisationsavoirs.fr/lubat/creolisation/JazzAfrique.pdf.

Citton, Yves. « Improvisation, rythmes et mondialisation. Quatorze thèses sur la fluidification sociale et les résistances idiorrythmiques », Rhuthmos, juillet 2010. www.rhuthmos.eu/spip.php?article22.

Coltrane, John. Je pars d'un point et je vais le plus loin possible. Entretiens avec Michel Delorme, suivis d'une lettre à Don deMichael, Éditions de l'éclat, 2011.

de Certeau, Michel. La culture au pluriel. Seuil, 1993.

Foucault, Michel. Le Courage de la vérité. Le gouvernement de soi et des autres II. Cours au Collège de France. 1984. Gallimard/Seuil, 2009.

---. Dits et écrits II. 1976-1988. Gallimard, 2001.

---. Le gouvernement de soi et des autres. Cours au Collège de France. 1982-1983. Gallimard/Seuil, 2008.

---. L’herméneutique du sujet. Cours au Collège de France. 1981-1982. Gallimard/Seuil, 2001.

---. Qu'est-ce que la critique ?/La culture de soi. Vrin, 2015.

Garcia, Renaud. Le désert de la critique. Déconstruction et politique. L'Échappée, 2015.

Gerber, Alain. Le cas Coltrane. Éditions Parenthèses, 1985.

---. « Le Cohelmec : l'improvisation en jazz moderne », in Beaud (Paul), Willener (Alfred) dir., Musique et vie quotidienne. Essai de sociologie d'une nouvelle culture. Mame, 1973, pp. 155-191.

---. « Le New Phonic Art : l'improvisation en musique contemporaine », in Beaud (Paul), Willener (Alfred) dir., Musique et vie quotidienne. Essai de sociologie d'une nouvelle culture. Mame, 1973, pp. 193-204.

Glissant, Édouard. La Cohée du Lamentin. Poétique V. Gallimard, 2005.

---. L'imaginaire des langues. Entretiens avec Lise Gauvin (1991-2009). Gallimard, 2010.

---. Introduction à une poétique du divers. Gallimard, 1996.

---. Philosophie de la Relation. Poésie en étendue. Gallimard, 2009.

---. Poétique de la relation. Poétique III. Gallimard, 1990.

---. Traité du Tout-monde. Poétique IV. Gallimard, 1997.

Glissant, Édouard et Alexandre Leupin. Les entretiens de Baton Rouge. Gallimard, 2008.

Goldmann, Lucien. La création culturelle dans la société moderne. Denoël, 1971. 
Granjon, Fabien. Les UZ-topies de Bernard Lubat - Dialogiques. Outre Mesure, 2016.

Hill, Stephanie. « "An Invitation for Disaster". Embracing the "Double Failure" of Improvisation », Critical Studies in Improvisation, vol. 9, no. 2, 2013. doi: http://dx.doi.org/10.21083/csieci.v9i2.2731.

Imbert, Raphaël. Jazz supreme. Initiés, mystiques et prophètes. Éditions de l'éclat, 2014.

Laborde, Denis. « Thelonious Monk, le sculpteur de silence ». L'Homme, no.158-159, 2001, pp. 139-178.

Lacan, Jacques. Le Séminaire (tome 7). L’éthique de la psychanalyse. Seuil, 1986.

Lachaud, Jean-Marc. Que peut (malgré tout) l'art ? L'Harmattan, 2015.

Lallement, Valérian et al. « Entretien avec André Minvielle ». Hermaphrodite, 2006. http://sitehermaphrodite.free.fr/article.php3?id_article=30

Lavaud, Patrick. « Bernard Lubat, un itinéraire. Entretien avec Bernard Lubat ». Amiras/Repères occitans, 1985, pp. 59-73.

Le Blanc, Guillaume. La philosophie comme contre-culture. PUF, 2014.

Levaillant, Denis. L'Improvisation musicale. Essai sur la puissance du jeu. Actes Sud, 1996.

Lubat, Bernard, intervention dans le séminaire de Marc Chemillier « Modélisation des savoirs musicaux relevant de l'oralité ». EHESS, 2010.

---. « Intervention au colloque "Improvisation : ordre et désordre” ». Festival Sons d’Hiver, janvier 2009, www.cielubat.org/images/stories/ecrivien/colloque.pdf.

Macé, Marielle. Styles. Critique de nos formes de vie. Gallimard, 2016.

Mansour, Leyla. Corps de guerre. Poétique de la rupture. L'Harmattan, 2012.

Ménil, Alain. Les voies de la créolisation. Essai sur Édouard Glissant. Saint-Vincent de Mercuze. De l'incidence éditeur, 2011.

Mittelberger, Annie-Claire et Jean-Pierre Zirn. [Film] Bernard Lubat, l'Amusicien, Mezzo/La Seine TV, 2012.

Moussaron, Jean-Pierre. Les blessures du désir. Pulsions et Puissances en Jazz. Alter ego éditions, 2012.

Nachmanovitch, Stephen. Free Play. The Power of Improvisation in Life and the Arts. Putnam Book, 1990.

Parker, William. « Intervention au colloque "Improvisation : ordre et désordre” ». Festival Sons d'Hiver, janvier 2009, www.cie-lubat.org/images/stories/ecrivien/colloque.pdf.

Patlotch. « Jazz et communisme », 2003, www. patlotch.free.fr/text/1e9b5431-3.html.

Pessoa, Fernando. Le livre de l'intranquillité. Christian Bourgois éditeur, 1999.

Quan Ninh, Lê. Improviser librement. Abécédaire d'une expérience. Mômeludies/CFMI de Lyon, 2010.

Rivière, Rémi. « Chao[s]opéra Tout-monde », Programme de la XXXème édition de l'hestejada de las arts. 2007, pp. 42-44. 
Saladin, Matthieu. Esthétique de l'improvisation libre. Expérimentation musicale et politique. Les Presses du réel, 2014.

Sawyer, R. Keith. « La conversation comme phénomène d'émergence collaborative ». Tracés, no.18, 2010, pp. 4567.

Sidran, Ben. Black Talk. Da Capo Press, 1971.

Siron, Jacques. La partition intérieure. Jazz, musiques improvisées. Outre Mesure, 2015.

Tassin, Étienne. «Qu'est-ce qu'un sujet politique ? Remarques sur les notions d'identité et d'action ». Esprit, no.230231, 1997, pp. 132-150.

Willener, Alfred. « Le groupe Faire : exploration, improvisation, construction », in Beaud, Paul et Willener, Alfred dir., Musique et vie quotidienne. Essai de sociologie d'une nouvelle culture. Mame, 1973a, pp. 207-244. 